\title{
Rapid Intensification of Typhoon Mujigae (2015) under Different Sea Surface Temperatures: Structural Changes Leading to Rapid Intensification
}

\author{
XIAOMIN CHEN \\ Key Laboratory for Mesoscale Severe Weather, Ministry of Education, and School of Atmospheric Sciences, \\ Nanjing University, Nanjing, China \\ Ming XUE \\ Key Laboratory for Mesoscale Severe Weather, Ministry of Education, and School of Atmospheric Sciences, \\ Nanjing University, Nanjing, China, and Center for Analysis and Prediction of Storms, and School of Meteorology, \\ University of Oklahoma, Norman, Oklahoma
}

\section{JUAN FANG}

Key Laboratory for Mesoscale Severe Weather, Ministry of Education, and School of Atmospheric Sciences, Nanjing University, Nanjing, China

(Manuscript received 13 January 2018, in final form 1 October 2018)

\begin{abstract}
The notable prelandfall rapid intensification (RI) of Typhoon Mujigae (2015) over abnormally warm water with moderate vertical wind shear (VWS) is investigated by performing a set of full-physics model simulations initialized with different sea surface temperatures (SSTs). While all experiments can reproduce RI, tropical cyclones (TCs) in cooler experiments initiate the RI $13 \mathrm{~h}$ later than those in warmer experiments. A comparison of structural changes preceding RI onset in two representative experiments with warmer and cooler SSTs (i.e., CTL and S1) indicates that both TCs undergo similar vertical alignment despite the moderate VWS. RI onset in CTL occurs $\sim 8 \mathrm{~h}$ before the full vertical alignment, while that in $\mathrm{S} 1$ occurs $\sim 5 \mathrm{~h}$ after. In both experiments precipitation becomes more symmetrically distributed around the vortex as vortex tilt decreases. In CTL, precipitation symmetricity is higher in the inner-core region, particularly for stratiform precipitation. All experiments indicate that RI onset occurs when the radius of maximum wind (RMW) contraction reaches a certain degree measured in terms of local Rossby number. The contraction occurs much earlier in CTL, leading to earlier RI. These results suggest that vertical alignment, albeit necessary, is not an effective RI indicator under different SSTs, while a more immediate cause of RI is the formation of a strong/ compact inner core with high precipitation symmetry. Diagnoses using the Sawyer-Eliassen equation indicate that in CTL the enhanced microphysical diabatic heating of additional midlevel and deep convection along with surface friction contribute to stronger boundary layer inflow near/inside the RMW, facilitating earlier RMW contraction.
\end{abstract}

\section{Introduction}

Rapid intensification (RI) of tropical cyclones (TCs) is usually an indispensable stage for a weak TC to develop into a devastating, strong hurricane/typhoon (e.g., Kaplan and DeMaria 2003; Chen et al. 2015). With ongoing global warming, TCs are tending to intensify more rapidly before landfall (Emanuel 2017), making the growing populations in coastal areas more vulnerable. Thus, accurately predicting (prelandfall) RI is highly desirable. However, over the past three decades, improvement in TC intensity forecasts, particularly for RI, has been slow

\footnotetext{
Corresponding author: Ming Xue, mxue@ou.edu
}

(DeMaria et al. 2014). Although favorable large-scale environmental conditions are important for RI occurrence, environmental factors alone are insufficient to differentiate RI and slowly intensifying cases (Hendricks et al. 2010). Studies in the recent decade have sought to identify RI precursors/indicators in terms of internal processes. Many of the precursors are discussed in the context of a sheared environment, as most RI cases are embedded in environments with moderate ${ }^{1}$ vertical wind

\footnotetext{
${ }^{1}$ Moderate VWS is defined as being within the 25 th and 75 th percentiles of the global VWS distribution $\left(4.5-11.0 \mathrm{~m} \mathrm{~s}^{-1}\right.$; RiosBerrios and Torn 2017).
} 
shear (VWS; mean value of 4-6 $\mathrm{m} \mathrm{s}^{-1}$ ) according to statistical analyses (Kaplan and DeMaria 2003; Kaplan et al. 2010).

Environmental VWS is a leading factor affecting TC intensity changes (DeMaria and Kaplan 1994; Kaplan and DeMaria 2003; Chen et al. 2015). Dynamically, VWS tilts a TC vortex from its upright position, weakening the TC primary circulation by inducing a wavenumber-1 asymmetry in vertical velocity and the distribution of precipitation (e.g., Jones 1995; Wang and Holland 1996; Corbosiero and Molinari 2002; Reasor and Montgomery 2015). The most intense convective precipitation shield (CPS) is located downshear to downshear left, where the midlevel vortex usually resides (Rappin and Nolan 2012; Zhang and Tao 2013; Rogers et al. 2015).

In a sheared environment, frequently discussed RI precursors related to TC structural changes include the vertical alignment of the tilted vortex and the increased azimuthal symmetry of precipitation. The two processes are not necessarily mutually exclusive. Both modeling and observational studies have indicated that as the midlevel vortex precesses into the upshear quadrants, when vertical alignment usually occurs, the downshear CPS rotates cyclonically into the same quadrants to form a closed eyewall (e.g., Zhang and Tao 2013; Rogers et al. 2015; Susca-Lopata et al. 2015; Munsell et al. 2017; Chen et al. 2018). These two processes help to reduce the wavenumber-1 asymmetry and to rebuild an enhanced, deep secondary circulation, which are beneficial to the upcoming RI occurrence. However, not all studies agree that vertical alignment is the cause of RI onset. In a numerical simulation of Hurricane Earl (2010), Chen and Gopalakrishnan (2015) found that the large vortex tilt decreased significantly after RI onset and thereby argued that the vertical alignment is the result, rather than the trigger, of RI onset.

Although the increased azimuthal symmetry of precipitation is considered favorable for RI occurrence, debates remain regarding which mode of precipitation plays a more important role in the onset of RI. One frequently investigated subject is convective bursts (CBs; intense and deep convection), which are responsible for RI occurrence (e.g., Reasor et al. 2009; Molinari and Vollaro 2010; Chen and Zhang 2013; Wang and Wang 2014; Harnos and Nesbitt 2016). Observational studies have found that the propagation of CBs from downshear to upshear prior to RI onset can help to distinguish the RI period from the slowly intensifying period (Stevenson et al. 2014; Rogers et al. 2016). However, the TC community has not reached a consensus regarding the importance of CBs to RI (Rogers 2010; McFarquhar et al. 2012; Hazelton et al. 2017). In a numerical simulation of Hurricane Dennis (2005),
Rogers (2010) found that RI onset is not led by an increase in the number or intensity of CBs; instead, an increasing low-level mass flux achieved by weaker updrafts was found to be the trigger of RI. Similarly, McFarquhar et al. (2012) and Hazelton et al. (2017) also found that the area/number of CBs did not increase until RI onset. These seemingly contradictory conclusions can be partially reconciled by considering the preferred radial location of CBs. Both real-case simulations (Hazelton et al. 2017) and composite analyses of airborne Doppler radar observations (Rogers et al. 2013) indicate that there are more CBs inside the radius of maximum wind (RMW) when the TC is going to intensify or is already intensifying, whereas CBs exist primarily outside the RMW during weakening and steady-state periods. Therefore, even if the number and intensity of CBs are fixed in time, the preferred radial location relative to the RMW can lead to discrepancies in the intensity change. The energy efficiency theory invoking the balanced vortex model predicts that the diabatic heating occurring in the region of high inertial stability inside the RMW can more effectively increase the potential energy of the vortex and intensify the balanced tangential winds (Schubert and Hack 1982; Pendergrass and Willoughby 2009).

In comparison, a series of parallel statistical studies using satellite datasets puts the emphasis on other modes of precipitation, ranging from shallow/moderate convection (Kieper and Jiang 2012; Zagrodnik and Jiang 2014; Tao and Jiang 2015; Alvey et al. 2015) to stratiform precipitation (Tao et al. 2017). These studies argued that the more azimuthally symmetric structures of these modes of precipitation, compared with CBs, play a more important role in RI onset. However, the coarse spatial and temporal resolutions inherent to satellite datasets limit their ability to capture the evolution of CBs that typically have short lifetimes of $\sim 1 \mathrm{~h}$ and small horizontal scales of $\sim 10 \mathrm{~km}$ (Montgomery et al. 2006; Houze et al. 2009), which leaves some uncertainty in the conclusions of previous studies related to the importance of CBs.

Despite the uncertainties associated with the two categories of RI precursors, the applicability of these precursors to various environments has been discussed in the literature. In an idealized ensemble study, Tao and Zhang (2014) found that vertical alignment is an effective indicator of RI onset, independent of varying VWS and SST. However, the relative importance of the various modes of precipitation to RI onset seems to vary with VWS magnitude - that is, as VWS magnitude increases, the contribution of CBs becomes more dominant compared with the other modes of precipitation (Harnos and Nesbitt 2011, 2016) — suggesting that at least part of the varying importance of the several modes of precipitation results from variability in their environments. 
(a)

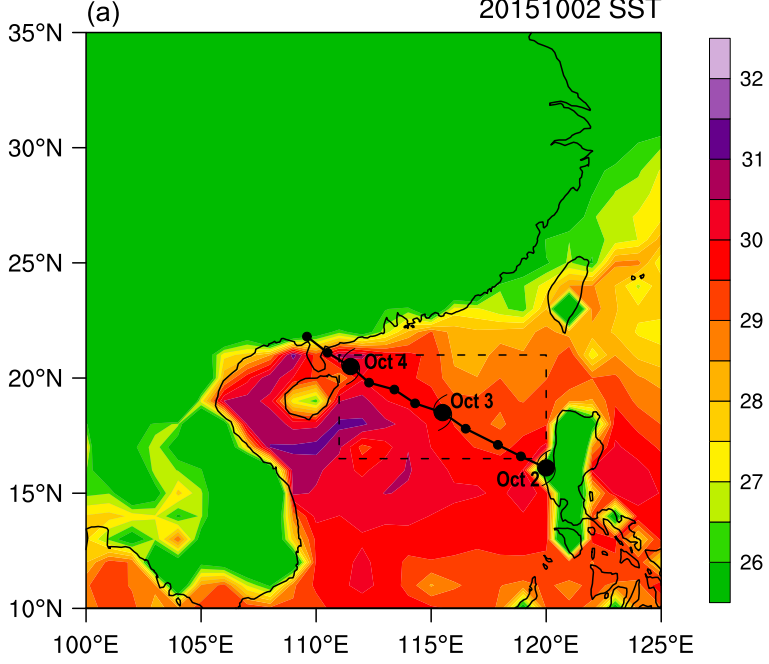

(b)
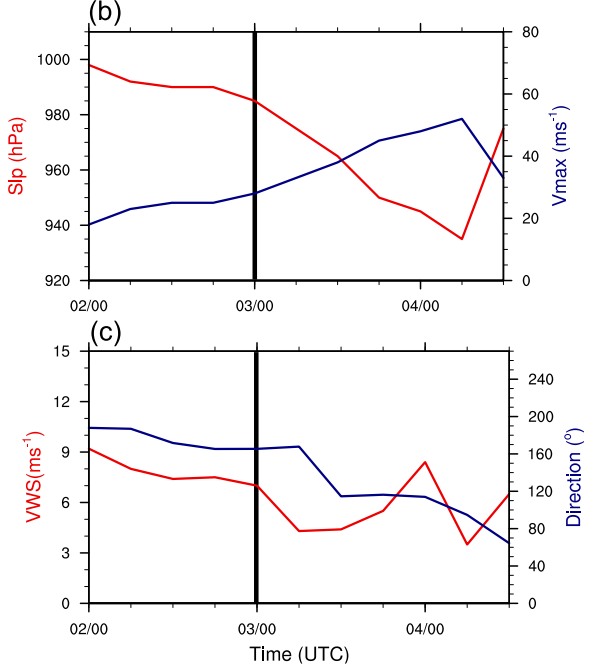

FIG. 1. (a) The surface skin temperature on 2 Oct (shading; ${ }^{\circ} \mathrm{C}$ ) and the 6-hourly best track from 0000 UTC 2 Oct to 1200 UTC 4 Oct 2015; (b) time series of the minimum SLP ( hPa) and maximum 10-m wind speed $\left(\mathrm{m} \mathrm{s}^{-1}\right)$ from 0000 UTC 2 Oct to 1200 UTC 4 Oct 2015; and (c) as in (b), but for the magnitude $\left(\mathrm{m} \mathrm{s}^{-1}\right)$ and heading $\left({ }^{\circ}\right.$; meteorological coordinate) of the 200-850-hPa environmental VWS. The black hurricane symbol in (a) marks location at 0000 UTC on each day. The thick vertical black lines in (b) and (c) indicate the time of RI onset.

To help reconcile discrepancies in interpreting the importance of RI precursors, we investigate in this study the inner-core processes leading to the RI onset of Typhoon Mujigae (2015) in the South China Sea (SCS) by performing a set of cloud-resolving, high-resolution numerical simulations under various SST conditions. The RI of Mujigae occurred in an environment with moderate VWS and abnormally high SST in the northern SCS. Because of the RI, it was upgraded directly from a severe tropical storm $^{2}$ to a supertyphoon just prior to its landfall in southern China, making it the globally costliest TC in that year, with an estimated $\$ 4.2$ billion loss for the Philippines and China (LeComte 2016).

Our goal is to investigate the key inner-core processes in terms of structural change leading to RI occurrence and, if possible, we will try to identify a more general RI indicator, applicable to a variety of SST conditions. Questions to be answered include the following: 1) Is vertical alignment a cause or a result of RI in this typhoon case study? 2) What is the relative importance of different modes of precipitation in triggering the onset of RI? 3) Does anomalously warm SST modulate certain modes of precipitation and alter the conclusions drawn for questions 1 and 2? If so, what is the key inner-core processes leading to RI onset? Do any other valuable RI indicators exist that can more effectively predict the timing of RI onset?

\footnotetext{
${ }^{2} \mathrm{TC}$ intensity grades are following the latest national standard proposed by the China Meteorological Administration (CMA) in 2006 .
}

The remainder of this paper is organized as follows. Section 2 provides an overview of the RI of Mujigae and the notable warm SST anomaly in the northern SCS. Section 3 describes the experiment design, the Advanced Research version of the Weather Research and Forecasting (WRF-ARW) Model setup and verification, and methods used in partitioning the various modes of precipitation. Section 4 analyzes the structural changes preceding RI onset, identifies a more general RI indicator applicable to different SST conditions, and diagnoses the dynamical mechanisms responsible for the difference in RI onset timing of TCs over warm and relatively cold sea surfaces. Finally, concluding remarks and a discussion are given in section 5 .

\section{Overview of Typhoon Mujigae (2015)}

\section{a. Rapid intensification of Typhoon Mujigae}

Typhoon Mujigae tracked across the northern Philiptppines and entered the SCS on 2 October 2015 (Fig. 1a). Afterward, it remained on a northwestward trajectory in the northern SCS. One day after it passed over warm seawater $\left(>29.5^{\circ} \mathrm{C}\right)$ and encountered a moderate $200-850-\mathrm{hPa}$ VWS $\left(7-9 \mathrm{~m} \mathrm{~s}^{-1}\right)$, it began to undergo RI (Fig. 1). Note that the 200-850-hPa VWS is calculated for the area between 200 and $800 \mathrm{~km}$ from the surface TC center. According to the best track datasets of the CMA, the RI of Mujigae lasted $30 \mathrm{~h}$ with a maximum sustained $10-\mathrm{m}$ wind (VMAX) increasing by $24 \mathrm{~m} \mathrm{~s}^{-1}$, exceeding the RI criteria of $15 \mathrm{~m} \mathrm{~s}^{-1}$ in $24 \mathrm{~h}$ proposed by 

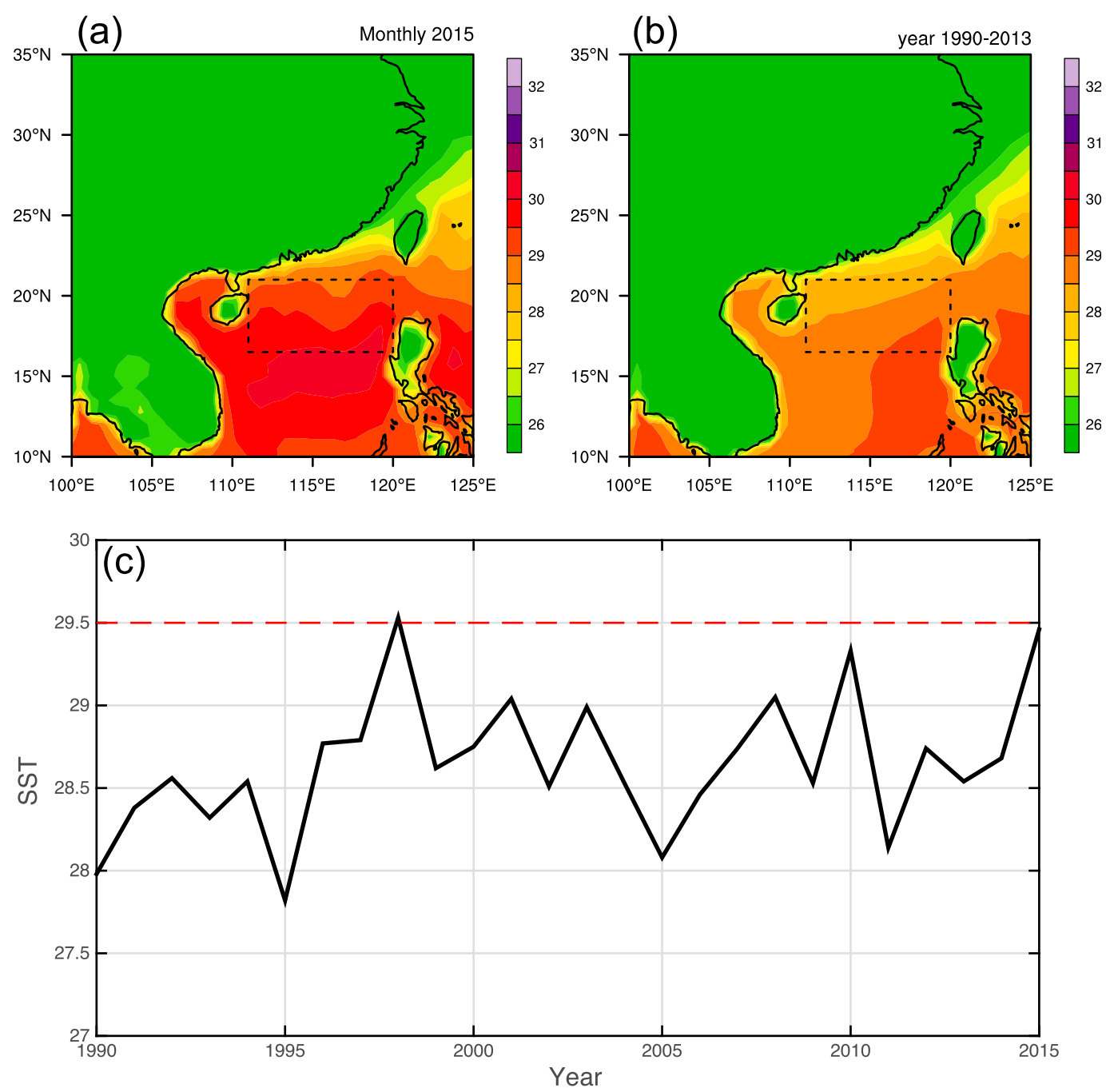

FIG. 2. (a) MMSST $\left({ }^{\circ} \mathrm{C}\right)$ averaged from 15 Sep to 15 Oct 2015; (b) as in (a), but averaged over 1990-2013; and (c) time series of MMSST averaged over the northern SCS [see rectangular box in (a) and (b)] from 1990 to 2015. The red dashed line in (c) denotes $29.5^{\circ} \mathrm{C}$. Skin temperature data are from the European Centre for Medium-Range Weather Forecasts interim reanalysis (ERA-Interim) datasets.

Kaplan and DeMaria (2003). The VMAX increased from $28 \mathrm{~m} \mathrm{~s}^{-1}$ (severe tropical storm intensity) at $0000 \mathrm{UTC}$ 3 October to $52 \mathrm{~m} \mathrm{~s}^{-1}$ (supertyphoon) at 0600 UTC 4 October, just prior to landfall in Guangdong Province, China. During the same period, the minimum sea level pressure (SLP) decreased from 985 to $935 \mathrm{hPa}$. Thus, Typhoon Mujigae became the strongest landfalling typhoon in Guangdong Province in October on record, according to CMA (2015). Note that the SST field and environmental VWS shown in Fig. 1 are derived from the NCEP Global Forecast System Final Analysis (GFS FNL) datasets.

\section{b. Anomalously warm SST in October}

One important factor responsible for the RI of Mujigae in the moderate VWS environment is the anomalously warm water (i.e., with maximum SST over $30^{\circ} \mathrm{C}$ ) in the northern SCS (Fig. 1a) at the time, which is much higher than the mean SST of RI cases in the region $\left(28.5^{\circ} \mathrm{C}\right)$ according to Chen et al. (2015). To further understand this warm SST anomaly, Figs. $2 a$ and $2 b$ show the monthly mean SST (MMSST; from 15 September to 15 October) in 2015 and the climatological MMSST averaged over 1990-2013. The 1-month period selected here for averaging is centered approximately on the RI period of Mujigae. The MMSST in 2015 in the northern SCS (black box in Fig. 2a) is $>1^{\circ} \mathrm{C}$ higher than the climatological mean (Fig. 2b). Note that the SST in the northern SCS on 2 October 2015 (Fig. 1) exhibits a similar pattern to the MMSST for this year (Fig. 2a), despite a more notable warming along the southern 


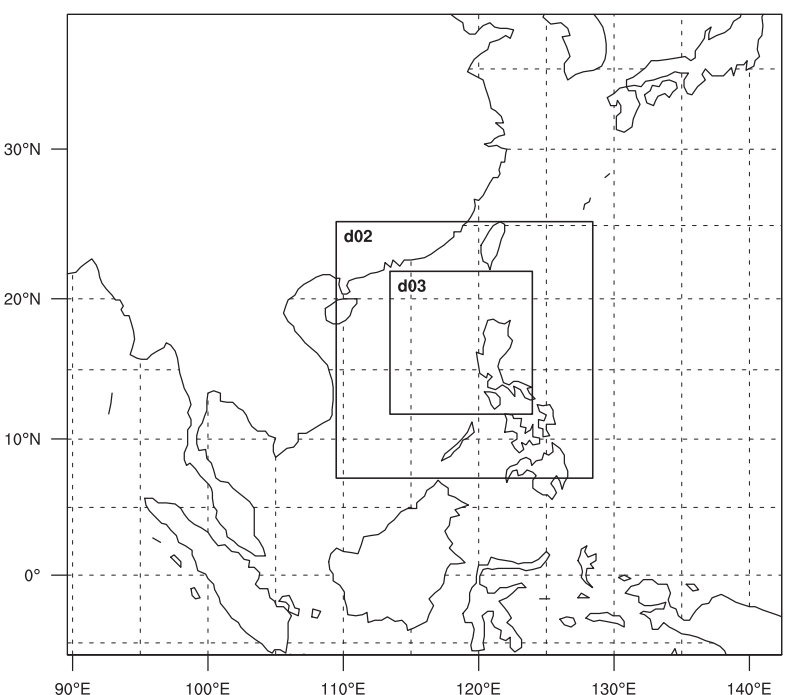

FIG. 3. Triply nested domain settings used for Typhoon Mujigae (2015) simulations.

coastline of China. The annual variation in MMSST averaged over the northern SCS (Fig. 2c) indicates that the MMSST in $2015\left(\sim 29.5^{\circ} \mathrm{C}\right)$ is the second warmest in this region since 1990 and only in a few years does MMSST notably exceed $29^{\circ} \mathrm{C}$ (i.e., years 1998,2010 , and 2015). This suggests that the exceptionally warm SST in the northern SCS may have played a prominent role in the RI of Mujigae. In the following discussion, we test this hypothesis by conducting a set of experiments with different SSTs.

\section{WRF simulation of Typhoon Mujigae (2015) and precipitation classification}

\section{a. Experiment design and model setup}

The WRF-ARW model, version 3.6 (WRF; Skamarock et al. 2008), is used in this study. Two-way interactive triple-nested domains are utilized with horizontal resolutions of 12,4 , and $1.33 \mathrm{~km}$, consisting of $466 \times 418$, $502 \times 502$, and $835 \times 838$ grid points, respectively (Fig. 3 ). The outermost domain is fixed, whereas the inner two domains move with the modeled TC. All three domains contain 51 sigma levels with the top level at $50 \mathrm{hPa}$. The outer two domains run from 0000 UTC 2 October to 1200 UTC 4 October, whereas the innermost domain is activated $12 \mathrm{~h}$ later and runs from 1200 UTC 2 October to 1200 UTC 4 October. In the control experiment (CTL), GFS FNL datasets are used for the initial and lateral boundary conditions. For simplicity, the SST is fixed at the initial conditions during the CTL simulation. To study the impact of the warm SST anomaly in the northern SCS, a set of sensitivity tests is conducted,
TABLE 1. Mujigae RI experiments and initial SST. Label "S" represents "sensitivity test."

\begin{tabular}{|c|c|}
\hline Expt & Description \\
\hline CTL & $\begin{array}{l}\text { Initialized with the SST at } \\
0000 \text { UTC } 2 \text { Oct } 2015\end{array}$ \\
\hline S1 & $\begin{array}{l}\text { Initialized with the 1990-2013 } \\
\text { MMSST }\end{array}$ \\
\hline $\mathrm{S} 1+50$ & $\begin{array}{l}\text { As in S1, but an extra } 50 \% \\
\text { of the } \mathrm{SST}_{\text {differences }} \\
\text { (i.e., } \mathrm{SST}_{\text {daily }}-\mathrm{MMSST} \text { ) } \\
\text { is added to the MMSST }\end{array}$ \\
\hline $\mathrm{S} 1+150$ & $\begin{array}{c}\text { As in } \mathrm{S} 1+50 \text {, but an extra } \\
150 \% \text { of SST differences } \\
\text { is added to the MMSST }\end{array}$ \\
\hline
\end{tabular}

wherein the SST at 0000 UTC 2 October is replaced by the climatological monthly mean state (S1), the climatological mean plus $50 \%$ of the warm SST anomaly (S1 + 50), and the climatological mean plus $150 \%$ of the warm SST anomaly $(\mathrm{S} 1+150)$, as detailed in Table 1 . Of note, the SST in CTL is equal to the climatological mean plus $100 \%$ of the warm SST anomaly. The SST averaged over the northern SCS (see the box in Fig. 2) for the four experiments is shown in Table 2. Other aspects of the model settings are kept the same among all the experiments.

The WRF Model physics configurations are as follows: the Kain-Fritsch cumulus parameterization scheme (Kain and Fritsch 1993) in the outermost domain; and the Thompson microphysical scheme (Thompson et al. 2008), the Mellor-Yamada-Janjić (MYJ) planetary boundary layer scheme (Mellor and Yamada 1982; Janjić 1990), the Dudhia shortwave radiation (Dudhia 1989), and the Rapid Radiative Transfer Model (RRTM) longwave radiation scheme (Mlawer et al. 1997) in all three domains.

\section{b. Verification of the CTL experiment}

Experiment CTL successfully reproduces the RI of Mujigae, as seen from the evolution of the minimum SLP and VMAX (Figs. 4a and 4b). Despite a somewhat faster intensification rate of the simulated TC after 0000 UTC 4 October, the timing of RI onset ${ }^{3}$ and the intensification rate during RI generally compare well with the CMA best track dataset. In addition, the simulated TC track matches the best track, and the landfall location is very close to observations (Fig. 4c). Note that the TC center is defined as the sea level pressure centroid in this study. Previous studies have suggested that

\footnotetext{
${ }^{3}$ In this study, RI onset is defined as the time when the increase in VMAX exceeds $15 \mathrm{~m} \mathrm{~s}^{-1}$ in the subsequent 24 -h or shorter period, if the RI duration is less than 1 day. An additional requirement is that the VMAX should increase in the first $6 \mathrm{~h}$ of the subsequent 24-h or shorter period.
} 
TABLE 2. Mean SST $\left({ }^{\circ} \mathrm{C}\right)$ averaged over the northern SCS (see rectangular box in Figs. $2 \mathrm{a}$ and $2 \mathrm{~b}$ ) for the four experiments.

\begin{tabular}{ccccc}
\hline \hline & CTL & S1 & S1 +50 & S1 + 150 \\
\hline SST & 29.6 & 28.6 & 29.1 & 30.1 \\
\hline
\end{tabular}

the pressure (or geopotential height) centroid is a better representation of the TC center, particularly for weak, loosely organized TCs (Nguyen et al. 2014; Chen et al. 2017).

To compare the simulated TC structure with observations, Figs. 5d-f show the evolution of the simulated layer-maximum ice-phase mixing ratio in the $8-10-\mathrm{km}$ height range, as the $91-\mathrm{GHz}$ polarization-corrected temperatures (PCT; Figs. 5a-c) reflect the reduced bright temperature associated with ice-phased particles above $8 \mathrm{~km}$ in height. The evolution of the simulated TC structure is generally comparable with observations. Prior to RI onset, the inner-core precipitation distribution exhibits a pronounced azimuthal wavenumber-1 asymmetry, with the most intense convection in the downshear-left (southeast) quadrant at 1700 UTC 2 October (Figs. 5a and 5d). Three hours later, a larger portion of the convective precipitation shield propagates into the upshear-left quadrant (Figs. 5b and 5e). During RI, both the simulated TC and observations indicate a small eye surrounded by a complete eyewall, with active outer spiral rainbands south of the TC center (Figs. 5c and 5f). A small discrepancy exists in that the simulated TC displays stronger convective activity in the northern part of the eyewall during this time (Figs. $5 \mathrm{c}$ and $5 \mathrm{f}$ ).
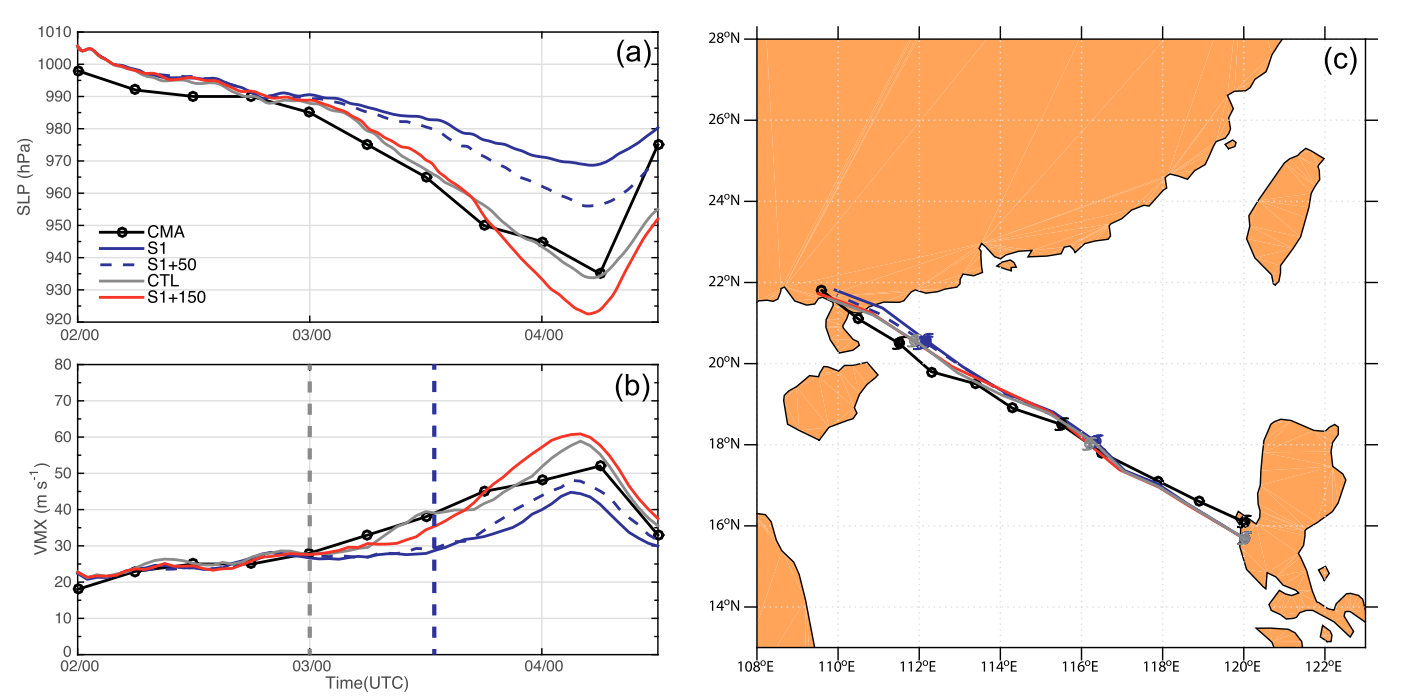

FIG. 4. Verification of simulated (a) minimum SLP (hPa), (b) 10-m maximum wind speed ( $\mathrm{m} \mathrm{s}^{-1}$ ), and (c) track. The black line in each panel represents the best track datasets of CMA. Experiments S1, S1 + 50, CTL, and S1 + 150 in each panel are denoted by solid blue, dashed blue, gray, and red lines, respectively. The RI onset timing for experiments $\mathrm{CTL}$ and $\mathrm{S} 1+150$ (S1 and S1 + 50) is marked as a vertical dashed gray (blue) line in (b).

\section{c. Classification of precipitation modes}

To examine the structure and evolution of different modes of precipitation within the TC inner core, we adopt the objective partitioning technique proposed by Rogers (2010) to divide the precipitation primarily into two distinct regimes, namely, convective and stratiform components. A supplementary regime is also included, labeled "other," and is applied when a grid point satisfies neither the stratiform nor convective criteria but does have precipitation $(<20 \mathrm{dBZ})$ at $3-\mathrm{km}$ height [for more details, see Rogers (2010)]. Based on results of the first partitioning step, we further divide the convective precipitation into different types of convection based on the height of the cloud top (Fritz et al. 2016): shallow cumulus (height $<$ $4 \mathrm{~km}$ ), midlevel convection ( $4<$ height $\leq 8 \mathrm{~km})$, and deep convection (height $>8 \mathrm{~km}$ ). The cloud top is represented by the $20-\mathrm{dBZ}$ echo top if the maximum reflectivity in a column is no less than $20 \mathrm{~dB} Z$. Otherwise, the $20-\mathrm{dBZ}$ echo-top height is set to zero. We also identify one type of extreme deep convection, namely $\mathrm{CBs}$, as discussed earlier. In this study, CBs belong to the large group of deep convection. Many different definitions of CBs have been used in previous literatures. In this study, we adopt the methods proposed by Rogers (2010) and Wang and Wang (2014) for comparison. Rogers (2010) defined a CB as a grid point where the layer-averaged vertical velocity within the 300-700-hPa layer exceeds $5 \mathrm{~m} \mathrm{~s}^{-1}$, whereas Wang and Wang (2014) defined a CB as a grid point where the vertical velocity at $12-\mathrm{km}$ height is $>7.5 \mathrm{~m} \mathrm{~s}^{-1}$. We obtained very similar results when using these two definitions of CBs. Thus, in the following analysis we 

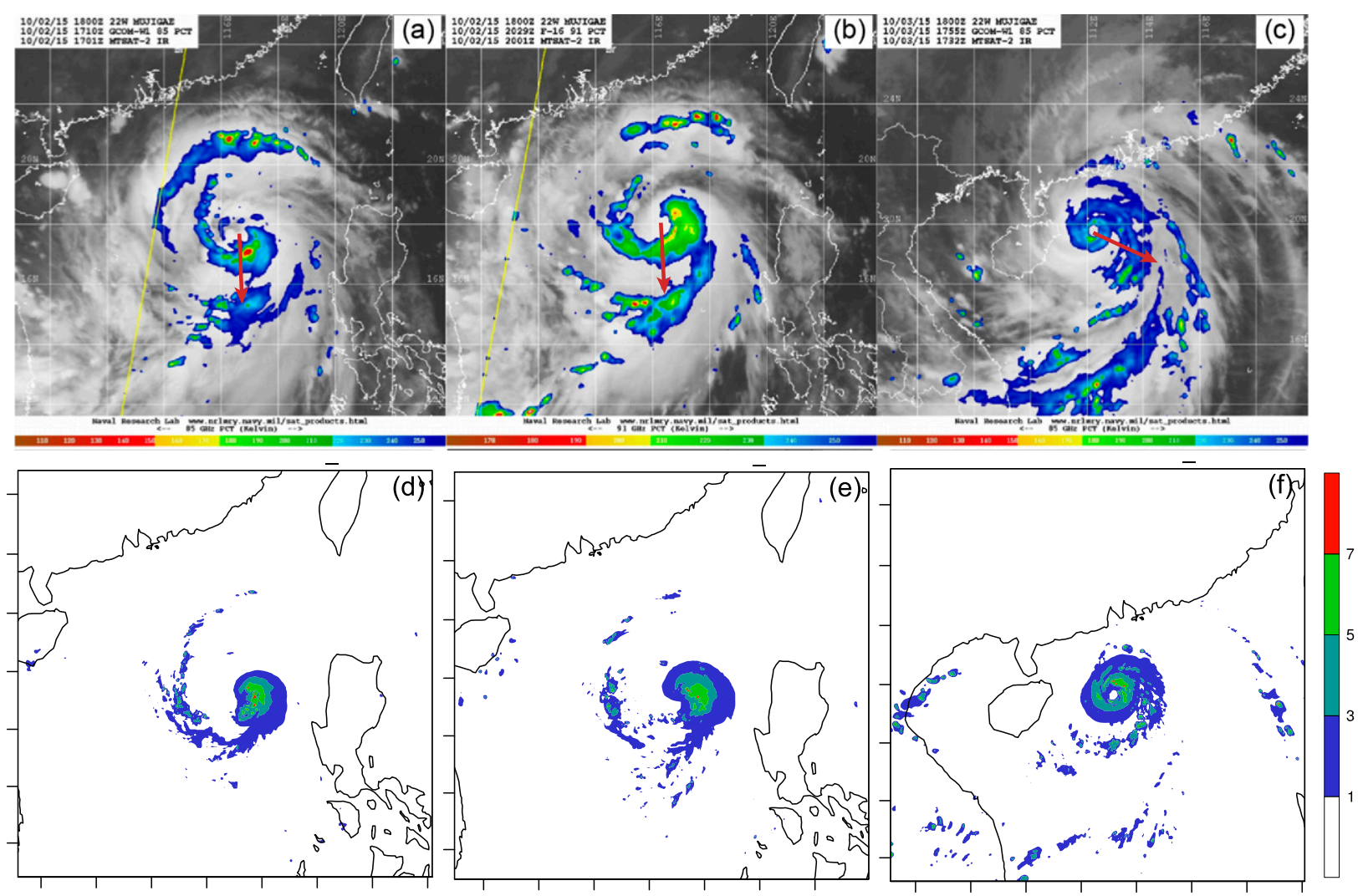

FIG. 5. (a)-(c) The 91-GHz PCT satellite microwave images (K) at 1700 UTC 2 Oct, 2000 UTC 2 Oct, and 1800 UTC 3 Oct, respectively. (d)-(f) The simulated layer-maximum ice-phase mixing ratio $\left(\mathrm{g} \mathrm{kg}^{-1}\right)$ at heights of $8-10 \mathrm{~km}$ and times corresponding to (a)-(c), respectively. Red arrows in (a)-(c) denote the 200-850-hPa environmental VWS.

show only the results obtained using the method proposed by Rogers (2010).

\section{Model results}

Figure 4 indicates that the warmer the sea surfaces in the northern SCS, the higher the maximum intensity of the simulated TCs, in agreement with the result of idealized simulations that involves no VWS (Črnivec et al. 2016). Experiment $\mathrm{S} 1+150$ has the maximum $\operatorname{VMAX}\left(61 \mathrm{~m} \mathrm{~s}^{-1}\right)$ and minimum SLP $(921 \mathrm{hPa})$. All simulated TCs in the four experiments undergo RI prior to landfall, while their RI onset timing is different. TCs over warm sea surfaces (CTL and S1 + 150) start to undergo RI at 0000 UTC 3 October, $\sim 13 \mathrm{~h}$ earlier than do TCs over relatively cold sea surfaces ( $\mathrm{S} 1$ and $\mathrm{S} 1+50)$. The RI duration of the $\mathrm{S} 1$ TC is $\sim 14 \mathrm{~h}$, as the VMAX of S1 TC increases from $29 \mathrm{~m} \mathrm{~s}^{-1}$ at 1300 UTC 3 October to $46 \mathrm{~m} \mathrm{~s}^{-1}$ at 0300 4 October. In the following analysis, we focus on the innercore processes that are responsible for the discrepancy in RI onset timing under different SST conditions by comparing two representative experiments with warm and relatively cold SST, namely, experiments CTL and S1.
Before examining the inner-core processes, we first examine the difference between the two experiments in terms of SST and related thermodynamic conditions in the lower boundary layer. Figure 6 shows the average SST values beneath the translating TCs and other related thermodynamic conditions in a central area within the radius of $120 \mathrm{~km}$ for CTL and S1 TCs. The initial SST difference between CTL and S1 (i.e., with the warm SST anomaly) is $\sim 0.6^{\circ} \mathrm{C}$, before it gradually decreases to $0.3^{\circ} \mathrm{C}$ by 1200 UTC 2 October with the translation of simulated TCs. This is followed by a steady increase throughout the RI period, reaching a value of $1.8^{\circ} \mathrm{C}$ by 0000 UTC 4 October. The higher SST in CTL contributes to an extra surface heat flux (SFX; including sensible and latent heat fluxes) of $\sim 100 \mathrm{~W} \mathrm{~m}^{-2}$ compared with $\mathrm{S} 1$ prior to 0400 UTC 3 October (Fig. 6b), resulting in a higher equivalent potential temperature $\theta_{e}$ in the boundary layer (Fig. 6c) and a stronger surface-based convective available potential energy (CAPE; Fig. 6d). However, because the difference in SST steadily increases with time along the TC track and CTL TC starts its RI much earlier, the SFX in CTL is enhanced much more quickly than that in S1 after 0400 UTC 3 October. The difference in the boundary layer 

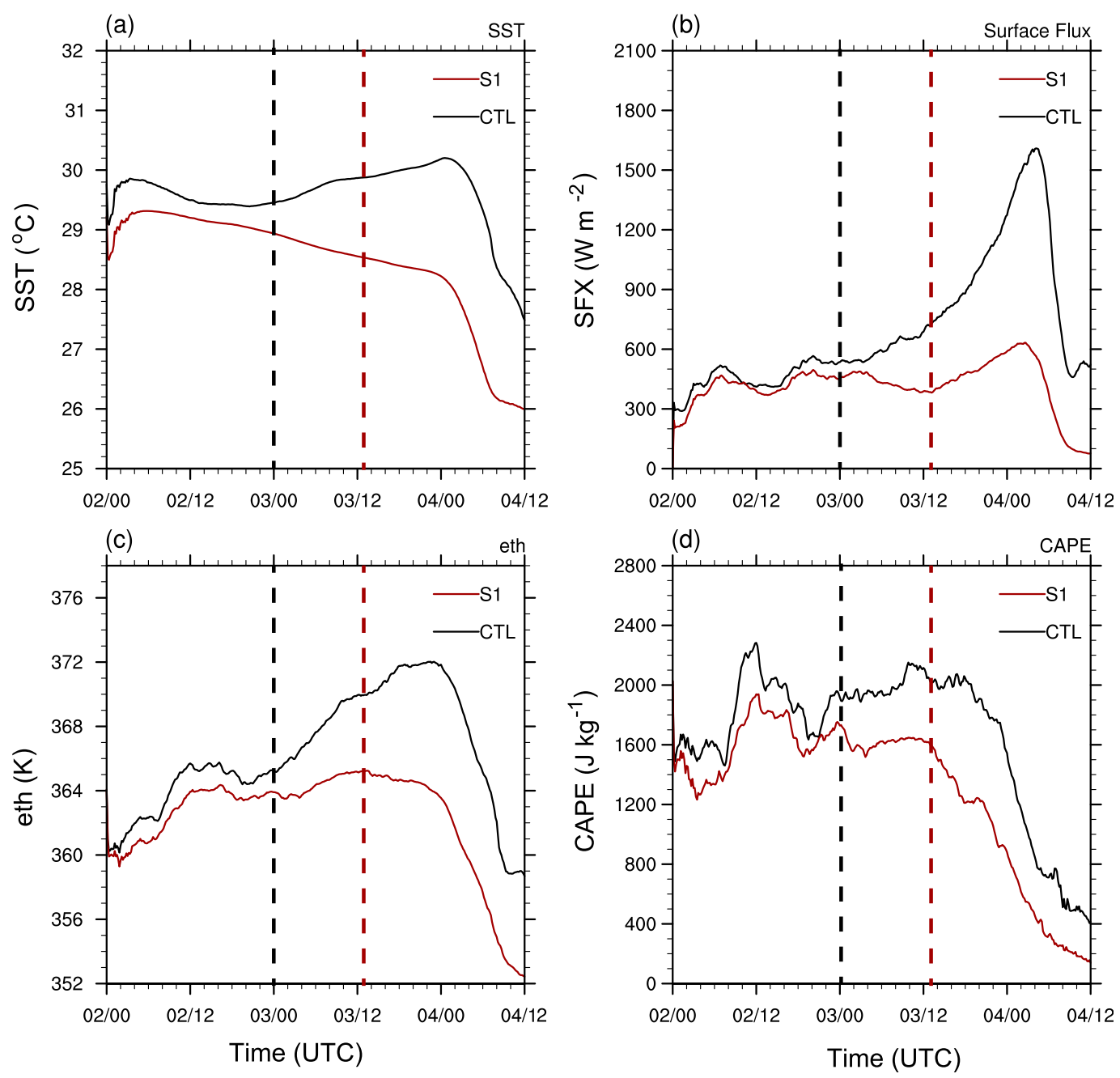

FIG. 6. Mean (a) SST $\left({ }^{\circ} \mathrm{C}\right)$, (b) surface heat fluxes $\left(\mathrm{W} \mathrm{m}^{-2}\right),(\mathrm{c}) \theta_{e}(\mathrm{~K})$ at the lowest model level, and (d) surfacebased CAPE $\left(\mathrm{J} \mathrm{kg}^{-1}\right)$ averaged in a circle of radius $120 \mathrm{~km}$ from the surface TC center. The solid black (red) line denotes the results of the CTL (S1) experiment. The dashed black (red) line denotes the RI onset time of the CTL (S1) TC.

$\theta_{e}$ between CTL and $\mathrm{S} 1$ thereby grows from $2 \mathrm{~K}$ at 0400 UTC 3 October to $9 \mathrm{~K}$ at 0000 UTC 4 October. During the same period, the difference in CAPE increases from $\sim 400$ to $\sim 900 \mathrm{~J} \mathrm{~kg}^{-1}$.

\section{a. Evolution of vortex structure in a sheared environment}

Prior to RI onset, the convective precipitation shield organized by VWS in both experiments resides initially in the downshear-left quadrant (Figs. 7a and 7f) and then rotates into the upshear flank (Figs. $7 \mathrm{~b}-\mathrm{e}$ and $7 \mathrm{~g}-\mathrm{j}$ ), increasing the precipitation symmetry in the inner-core region. Concurrently, the $450-850-\mathrm{hPa}$ TC vortex tilt in both experiments shrinks substantially after the 450-hPa TC vortex precesses into the upshear flank. Of note, the TC vortex tilt is defined as the difference between the $850-$ and $450-\mathrm{hPa}$ geopotential height centroids. These two concurrent events are the primary RI precursors, as discussed in section 1 . The magnitude of the VWS in the two experiments is similar throughout the integration period (not shown). Figure 8a further shows that the evolution of the tilt vector in the two experiments is quite similar: the midlevel TC vortex in CTL and S1 precesses from downshear left to upshear at the same time ( $\sim 1400$ UTC 2 October). A small discrepancy lies in the magnitude of the vortex tilt, as the S1 TC has a relatively larger tilt $(<10 \mathrm{~km})$ than the CTL TC. Both TCs become fully vertically aligned (i.e., the magnitude of tilt is close to zero) at 0800 UTC 3 October, while RI onset in CTL occurs $\sim 8 \mathrm{~h}$ before that time and that in S1 occurs $\sim 5 \mathrm{~h}$ after that time. These results suggest that 


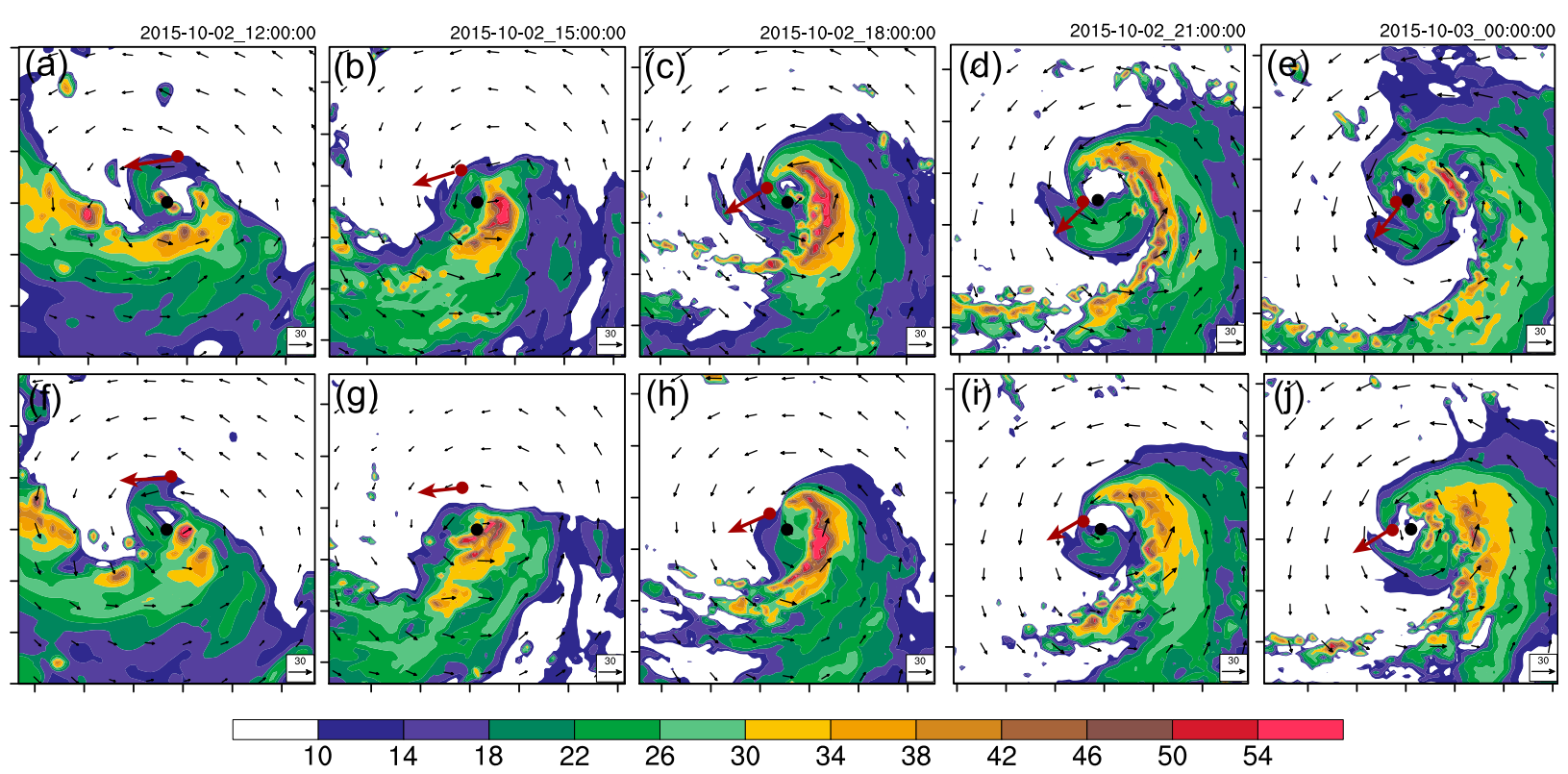

FIG. 7. Evolution of 450-hPa simulated radar reflectivity (shading; dBZ) and storm-relative winds (vectors; $\mathrm{m} \mathrm{s}^{-1}$ ) from $1200 \mathrm{UTC} 2 \mathrm{Oct}$ to 0000 UTC 3 Oct at 3-h intervals for (a)-(e) CTL and (f)-(j) S1. The thick red arrow in each panel denotes the 450-850-hPa environmental VWS. The red (black) dot indicates the 850- (450-) hPa TC center. The domain size of each panel is $3^{\circ} \times 3^{\circ}$.

vertical alignment alone is insufficient to predict RI occurrence.

We further examine the evolution of the inner-core structure in both experiments (Figs. $8 \mathrm{~b}$ and $8 \mathrm{c}$ ). The difference in the maximum azimuthal-mean tangential wind $v_{m}$ at $10-\mathrm{m}$ height between the CTL and S1 TCs is rather small prior to 0600 UTC 3 October. However, differences in the radius of $v_{m}$ (i.e., RMW) appear from 1800 UTC 2 October. The RMW of the CTL TC steadily contracts inward, whereas the RMW of the S1 TC does not exhibit a contraction until 1200 UTC 3 October (i.e., $1 \mathrm{~h}$ before the RI onset of the S1 TC). To quantify the differences in the TC inner-core structure and intensity, Fig. 8c shows the evolution of the local Rossby number at $10-\mathrm{m}$ height. The local Rossby number is defined as Ro $=v_{m} /\left(r_{m} f\right)$, where $f$ and $r_{m}$ represent the Coriolis parameter at the TC center and the RMW, respectively. Interestingly, in both experiments RI onset occurs at the time when Ro exceeds 12, and the increase in Ro is primarily due to RMW contraction. The other two experiments (i.e., $\mathrm{S} 1+50$ and $\mathrm{S} 1+150$ ) exhibit the same phenomenon (not shown). Note that Ro $=12$ is similar to the ensemble-mean value for RI groups (14) reported by Miyamoto and Nolan (2018), although they calculated Ro above the boundary layer (i.e., $z=2 \mathrm{~km}$ ) and invoked the argument of Ekman pumping in the boundary layer. Our results indicate that the RMW contraction at $10 \mathrm{~m}$ or lower boundary layer occurs $3 \mathrm{~h}$ earlier than that above the boundary layer (i.e., $z=$ $2 \mathrm{~km})$. This can be understood as surface friction decelerates the tangential momentum most notably in the lower boundary layer, which induces stronger boundary layer inflow and may cause earlier RMW contraction in the lower boundary layer, if contraction happens. These suggest that Ro at $10-\mathrm{m}$ height is a better indicator of RI occurrence. The above analysis indicates that in addition to vertical alignment, the RI occurrence requires the TC inner core to be intense and small enough.

Given that the evolution of TC inner-core structure is fundamentally connected to condensational diabatic heating (e.g., Pendergrass and Willoughby 2009), we next analyze the evolution of various modes of precipitation in section $4 \mathrm{~b}$ and identify the key modes of precipitation responsible for the earlier contraction of the RMW for the CTL TC in section 4c.

\section{b. Modes of precipitation prior to RI onset}

Figures $9 \mathrm{a}$ and $9 \mathrm{~b}$ show the simulated reflectivity at $850 \mathrm{hPa}$ and the region with averaged vertical velocity in the $0.9-2.1-\mathrm{km}$ layer exceeding $0.5 \mathrm{~m} \mathrm{~s}^{-1}$ at 1800 UTC 2 October for both experiments. According to the definition of convective precipitation of Rogers (2010), the region with black contours of vertical velocity is treated as convective precipitation. A comparison of Figs. 9c and 9d with Figs. 9a and 9b reveals that the convective-stratiform partitioning method reasonably separates the convective and stratiform components. We also compared the vertical structures of convective and stratiform components with those from 

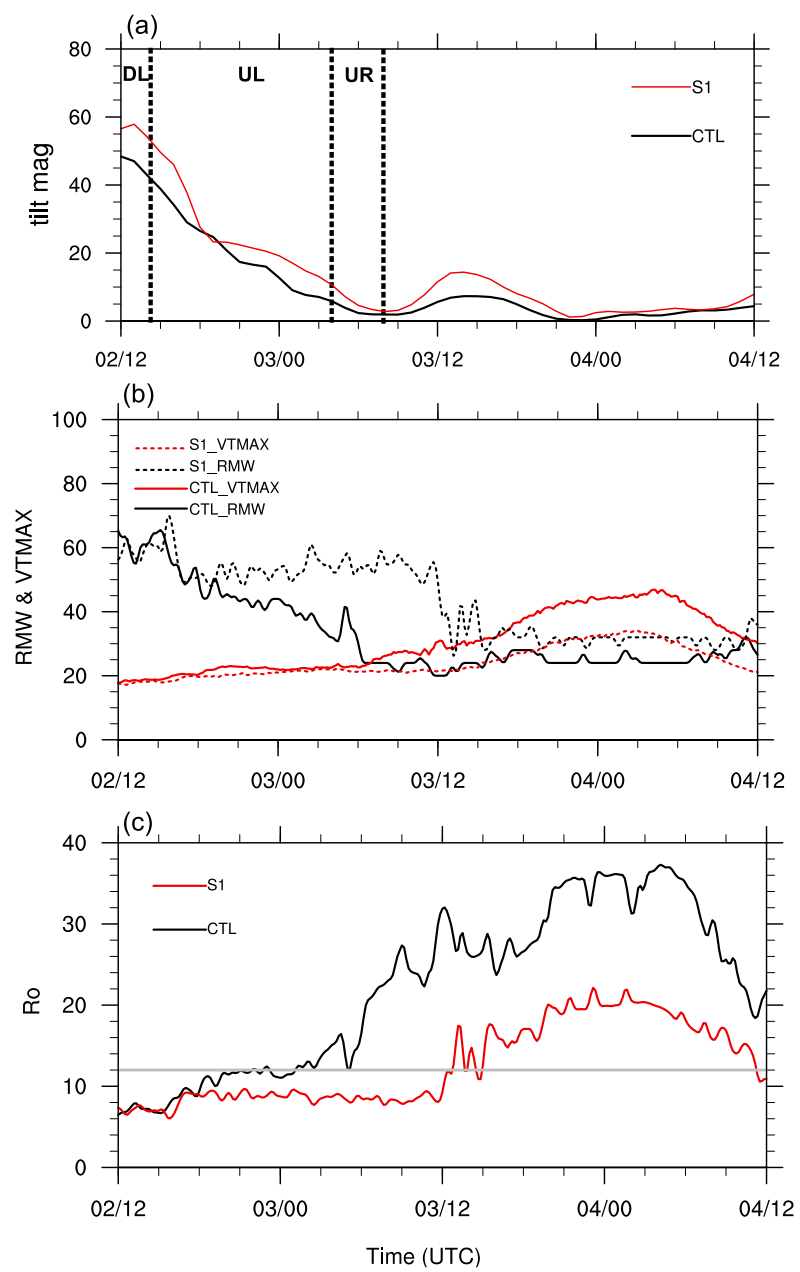

FIG. 8. (a) Evolution of the magnitude of vortex tilt (km) for the CTL (black line) and S1 (red line) TCs from 0000 UTC 2 Oct to 1200 UTC 4 Oct. The three dashed lines mark the downshear-left (DL), upshear-left (UL), and upshear-right (UR) quadrants. (b) Evolution of the RMW (black; km) and $v_{m}$ (red; $\mathrm{m} \mathrm{s}^{-1}$ ) at $10-\mathrm{m}$ height for the CTL (solid) and S1 (dashed) TCs from 1200 UTC 2 Oct to 1200 UTC 4 Oct. (c) As in (b), but for Ro at 10-m height. The solid gray line in (c) denotes Ro $=12$.

Rogers (2010), and found similar structures in terms of vertical velocity and reflectivity (not shown). Evidently, the CTL TC has a larger coverage area of stratiform precipitation, particularly in the upshear quadrants at this time. Figures $9 \mathrm{e}$ and $9 \mathrm{f}$ further show that in both experiments, the convective region primarily consists of deep convection, with CBs, identified according to the definition of Rogers (2010), located in the central region of deep convection. In short, the partitioning methods employed here effectively separate the various modes of precipitation.

To investigate the RI precursors in terms of the various modes of precipitation, the evolution of the percentage of their corresponding grid points within $120 \mathrm{~km}$ from the surface TC center is shown in Fig. 10. Prior to 3 October, the percentage of stratiform grid points in both experiments gradually increases with time, and the CTL TC has $10 \%-15 \%$ more stratiform points than the S1 TC. However, the percentage of the convective points, particularly the shallow and deep convection (including CBs), for the CTL and S1 TCs are comparable, and the shallow convection accounts for the smallest ratio of convective precipitation (Figs. 10a and 10b). Of note, the CBs account for a small ratio $(<10 \%)$ of the deep convection (Fig. 10c).

The percentage of deep convection in the CTL TC does not show a steady increasing trend until its RI onset (i.e., 0000 UTC 3 October), consistent with the results of previous studies (e.g., McFarquhar et al. 2012; Hazelton et al. 2017). In contrast, the percentage of deep convection in the S1 TC shows an increasing trend $6 \mathrm{~h}$ earlier than the RI onset of the S1 TC (i.e., 1300 UTC 3 October), followed by a decreasing trend after 1800 UTC 3 October as the SST decreases. The evolution of midlevel convection prior to RI onset in both experiments shows a similar trend: a notable increase in the areal coverage within the central area $(r=120 \mathrm{~km})$ precedes the RI onset of both the CTL and S1 TCs, despite the higher percentage of midlevel convection for the CTL TC. However, the areal coverage of midlevel convection of the S1 TC also increases during the period 1300-1800 UTC 2 October, one day earlier than its RI onset. These results suggest that it is difficult to define a consistent RI precursor based on the areal coverage of various modes of precipitation.

Figure 10d further shows the evolution of symmetricity ${ }^{4}$ $P_{s}$ of convective and stratiform precipitation within the radius of $60 \mathrm{~km}$ (i.e., inside the RMW at 1200 UTC 2 October), both of which gradually increase prior to 0900 UTC 3 October. Special attention is paid to the period from 1200 UTC 2 October to 0000 UTC 3 October, as it corresponds to the time when the differences in the RMW start to appear. The CTL TC has notably higher $(\sim 0.2)$ stratiform precipitation symmetricity than that of the S1 TC during this period, highlighting the close relationship between higher stratiform precipitation symmetricity and RI onset as in Tao et al. (2017). In comparison, the convective precipitation symmetricity of the CTL TC is only slightly higher. Statistical results indicate that the CTL TC has more points of deep and

\footnotetext{
${ }^{4}$ The precipitation symmetricity is examined by separating the storm-relative structure into eight equal regions, each with $45^{\circ}$ of azimuthal coverage. If the area coverage for a specific type of precipitation in a region exceeds $20 \%$, the region is recorded as having this type of rainfall. Precipitation symmetricity is defined as $P_{s}=n / 8$, where $n$ is the number of regions with rainfall.
} 

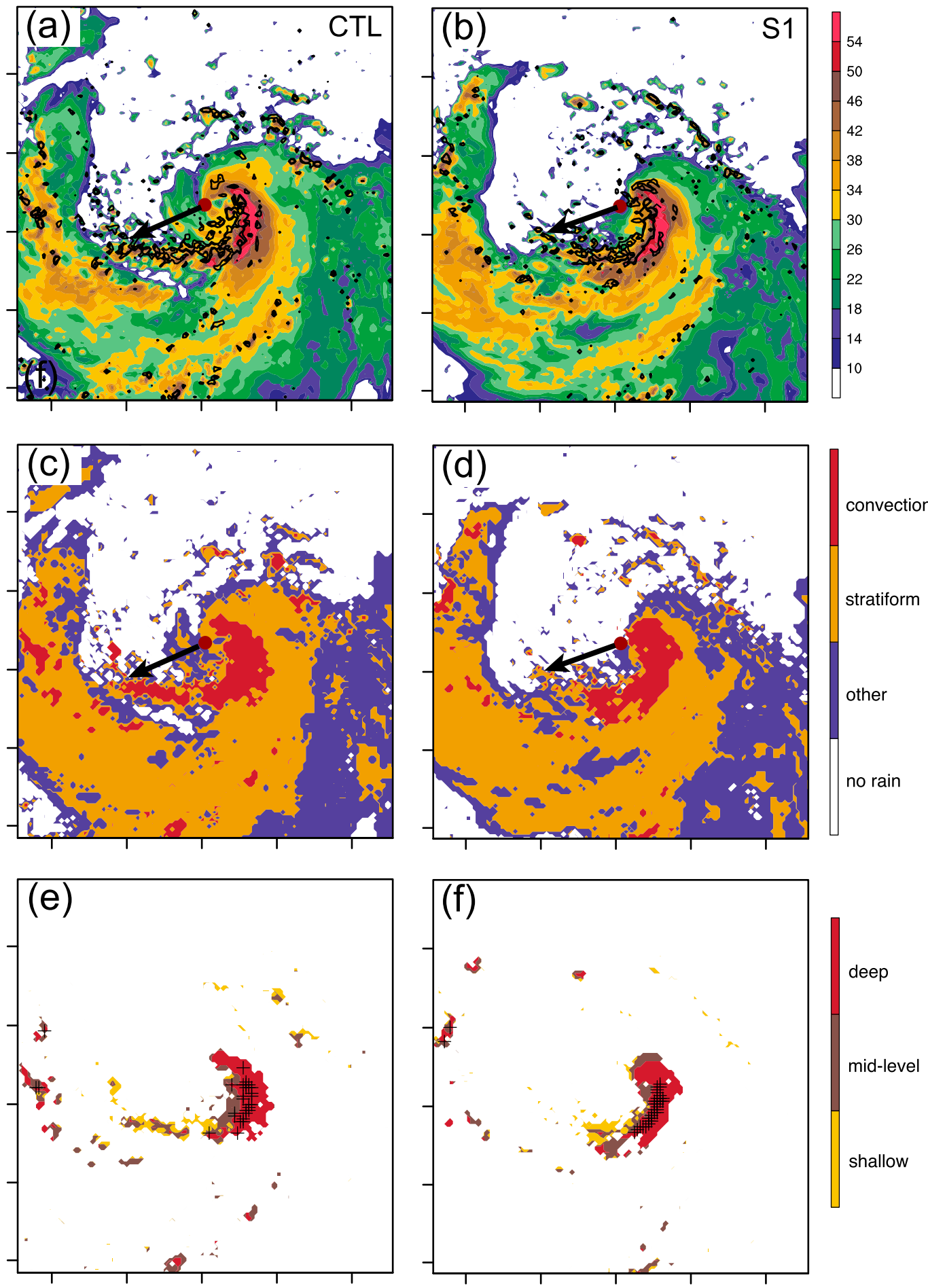

FIG. 9. (a),(b) Simulated radar reflectivity (shading) at $850-\mathrm{hPa}$ and averaged vertical velocity (contours at 0.5 and $1 \mathrm{~m} \mathrm{~s}^{-1}$ ) in the 0.9-2.1-km layer for 1800 UTC 2 Oct. (c),(d) Results of the convective-stratiform partitioning method. (e),(f) Results of partitioning of various types of convection (shading), overlain with CBs (+). Columns show results for (left) CTL and (right) S1. The thick black arrow in (a)-(d) represents the 200-850-hPa VWS. The domain size of each panel is $5^{\circ} \times 5^{\circ}$. 

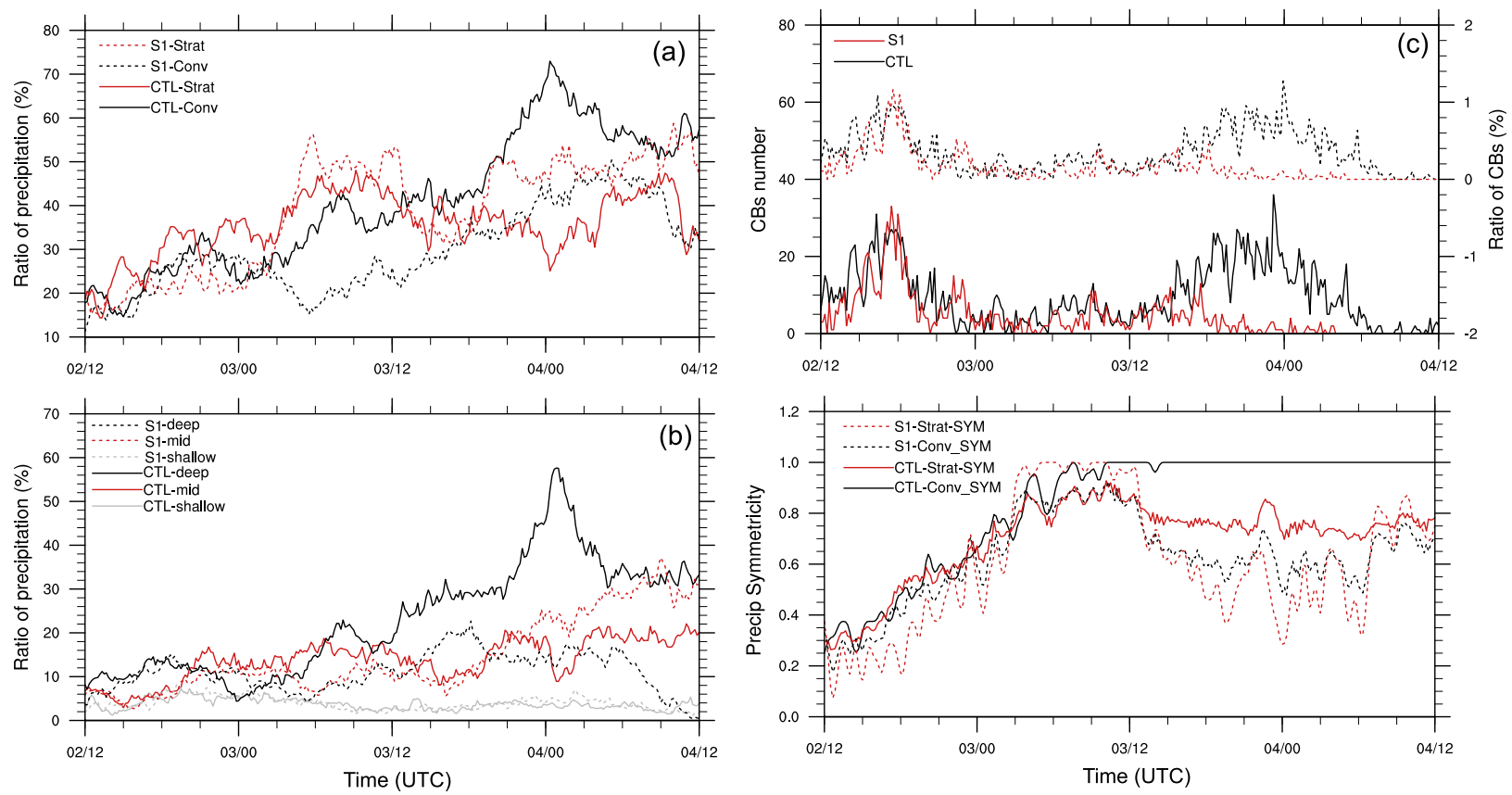

FIG. 10. (a) Evolution of the percentage of convective (black) and stratiform (red) grid points within $120 \mathrm{~km}$ of the surface TC center for experiments CTL (solid line) and S1 (dashed line). (b) As in (a), but for the percentage of shallow (gray), midlevel (red), and deep convection (black) grid points within the same area. (c) As in (a), but with the number (solid line) and percentage (dashed line) of CBs for experiments CTL (black) and S1 (red). (d) As in (a), but for the precipitation symmetricity for stratiform (red) and convective (black) precipitation within $60 \mathrm{~km}$ of the surface TC center.

midlevel convection (i.e., 1533 and 2951, respectively) than the S1 TC during the 12 -h period (see Table 3), and the additional points are located primarily near/inside the RMW (not shown), suggesting that the diabatic heating in the inner-core region of the CTL TC may be stronger than that of the S1 TC.

To test this hypothesis, Fig. 11 shows the microphysical diabatic heating of various modes of precipitation averaged within the radius of $60 \mathrm{~km}$. The stratiform precipitation has a negligible contribution to the areally averaged diabatic heating during the 12-h period (Figs. 11c and 11f). In contrast, the convective precipitation is the dominant heating source within the inner-core region (Figs. 11a and 11b and Figs. 11d and 11e), which is primarily made up of deep and midlevel convection (Figs. 11g and 11h and Figs. $11 \mathrm{j}$ and $11 \mathrm{k}$ ). The more active midlevel and deep convection contribute to the stronger diabatic heating inside the RMW of the CTL TC.

Figure 12 shows the radius-height distribution of diabatic heating of different types of precipitation as well as their differences between the CTL and S1 TCs. The azimuthal-mean microphysical diabatic heating rate of the CTL TC is $1-2 \mathrm{~K} \mathrm{~h}^{-1}$ higher than that of the S1 TC inside the RMW (Fig. 12c). The azimuthalmean diabatic heating of stratiform is much smaller compared with that of convective precipitation, with the peak stratiform diabatic heating $\left(<2 \mathrm{~K} \mathrm{~h}^{-1}\right)$ at around $9-10 \mathrm{~km}$ between $r=100$ and $150 \mathrm{~km}$. A closer investigation of both experiments reveals that the convective diabatic heating near the RMW is from deep and midlevel convection (Fig. 13), whereas the azimuthal-mean diabatic heating of $\mathrm{CBs}$ is maximized in the $6-9-\mathrm{km}$ layer inside the RMW, accounting for $30 \%-40 \%$ of the diabatic heating of deep convection (Figs. $13 \mathrm{j}$ and $13 \mathrm{k}$ ). Thus, it is the overall contribution of deep convection, rather than that of CBs alone, that dominates the total diabatic heating near the RMW.

In short, the above analyses indicate that the CTL TC has a higher precipitation symmetricity, particularly for stratiform precipitation, preceding RI onset, while it is the more active midlevel and deep convection inside the RMW that lead to stronger diabatic heating.

\section{c. What is responsible for the earlier RMW contraction of the CTL TC?}

As discussed in section 4a, the earlier RI onset of the CTL TC is closely related to its earlier RMW contraction than seen for the S1 TC. During RMW contraction, tangential winds inside the RMW intensify more 
TABLE 3. Number of midlevel, deep convection, and total points within the circle with a radius of $120 \mathrm{~km}$ during the period between 1200 UTC 2 Oct and 0000 UTC 3 Oct in 10-min intervals. The numbers in parentheses represent the percentage of the differences between CTL and S1 to the total number of points.

\begin{tabular}{lccc}
\hline \hline & Midlevel & Deep & Total \\
\hline CTL & 18381 & 22605 & 206608 \\
S1 & 15430 & 21072 & 206529 \\
CTL - S1 & $2951(1.43 \%)$ & $1533(0.74 \%)$ & 79 \\
\hline
\end{tabular}

rapidly than do those at the RMW (Stern et al. 2015), which is attributed to the secondary circulation that advects absolute angular momentum surfaces inward. Therefore, we first compare the mean secondary circulation between the two experiments to identify their differences and then analyze the mechanisms responsible for these differences in this subsection. The averaged period is the same as that used in Figs. 12 and 13 .
Figures $14 \mathrm{a}$ and $14 \mathrm{c}$ show that the CTL TC has a stronger upward branch of secondary circulation than the S1 TC. The maximum difference in azimuthal-mean vertical motion is $\sim 0.06 \mathrm{~m} \mathrm{~s}^{-1}$ in the mid- to upper troposphere (see Fig. 14e), whereas the difference in azimuthal-mean radial inflow in the boundary layer is maximized $\left(\sim 0.8 \mathrm{~m} \mathrm{~s}^{-1}\right)$ near a $50-\mathrm{km}$ radius. The upper-tropospheric outflow of the CTL TC is $\sim 3 \mathrm{~m} \mathrm{~s}^{-1}$ larger than that of the $\mathrm{S} 1 \mathrm{TC}$. The primary circulation of the CTL TC is stronger and higher than that of the S1 TC, particularly inside the RMW (Figs. 14b, 14d, and 14f). In addition, the strengthened diabatic heating near/ inside the RMW of the CTL TC (Figs. 14b, 14d, and 14f) is collocated with the enhanced secondary circulation (Fig. 14e). Considering these results, it is hypothesized that the discrepancies in the axisymmetric microphysical diabatic heating near/inside the RMW contributes to the differences in the secondary circulation, which may further impact the RMW contraction and TC intensity.
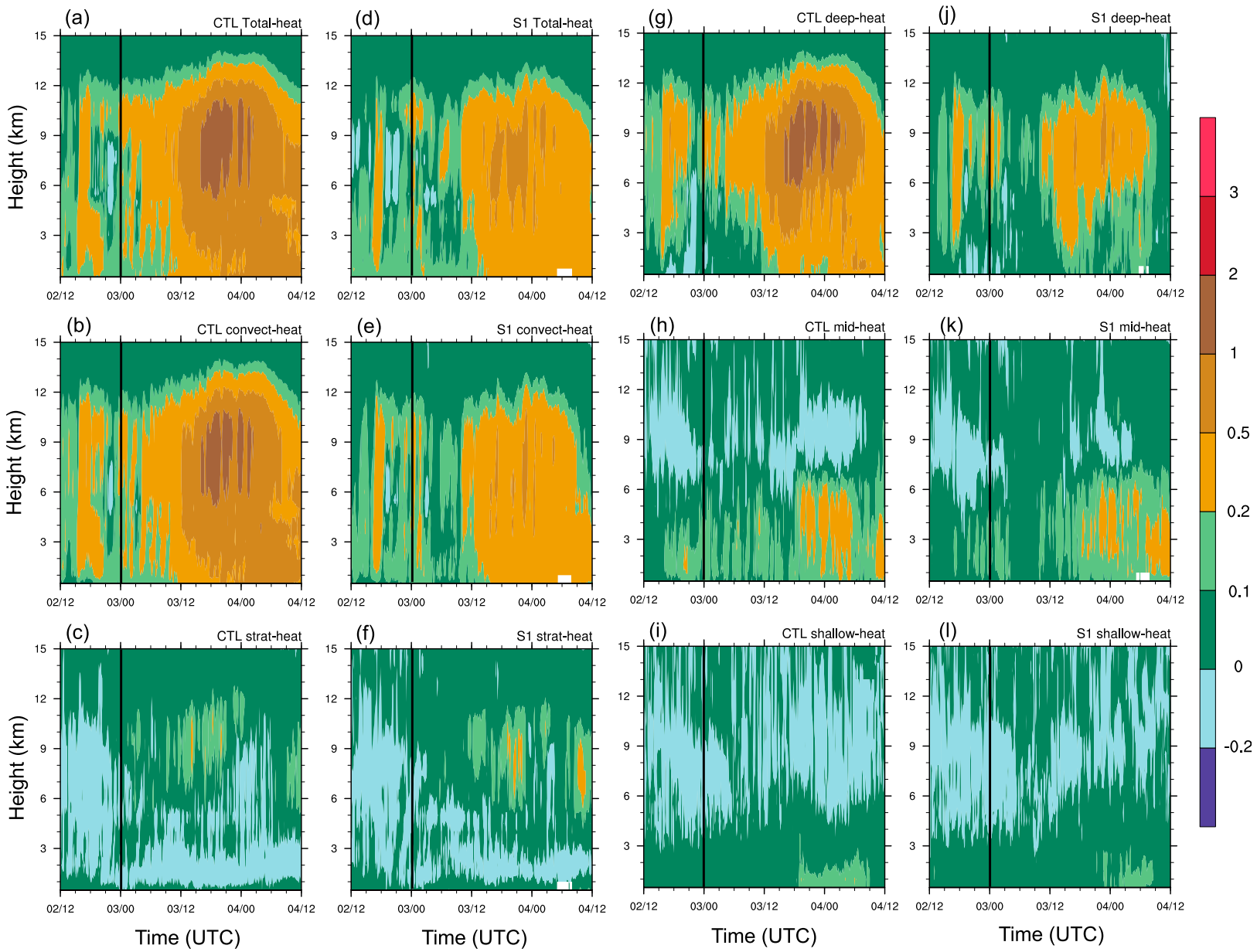

FIG. 11. Time-height diagram for the diabatic heating rate $\left(10^{4} \mathrm{~K} \mathrm{~h}^{-1}\right)$ of (a),(d) total, (b),(e) convective, (c),(f) stratiform, (g),(j) deep, (h),(k) midlevel, and (i),(l) shallow convection points within a radius of $60 \mathrm{~km}$ of the TC center. Columns relate to (a)-(c),(g)-(i) the CTL TC and (d)-(f), (j)-(i) the S1 TC. The solid black line in each panel is the RI onset timing of the CTL TC. 

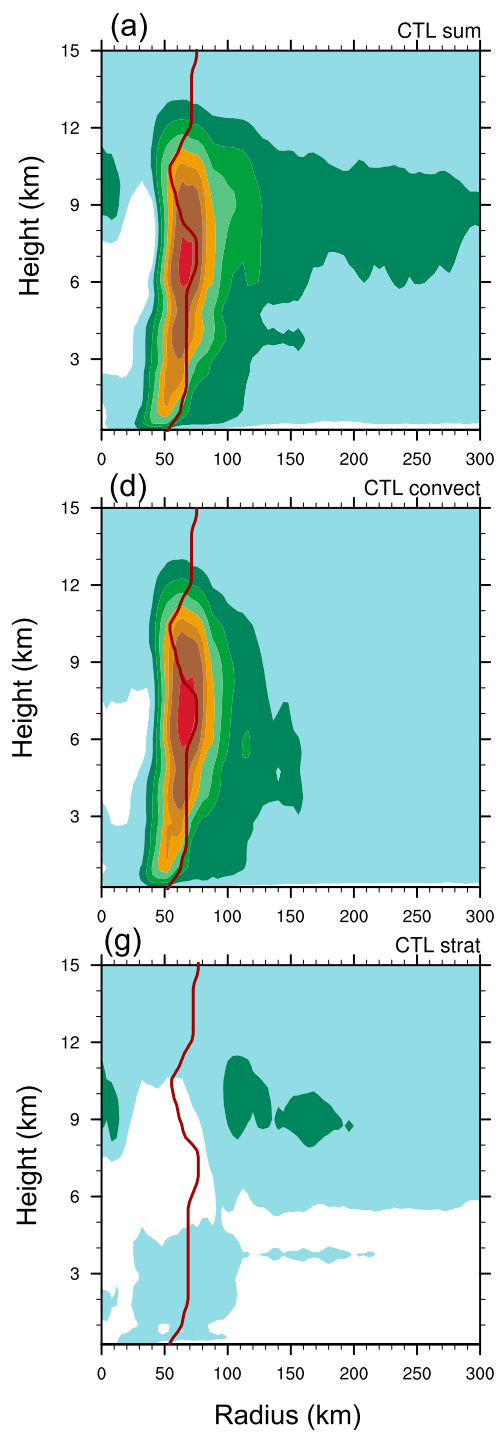

(b)

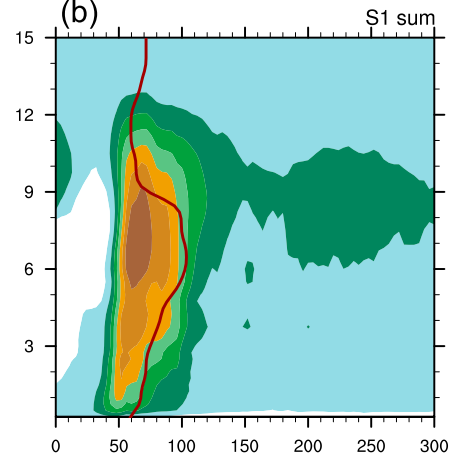

(e)
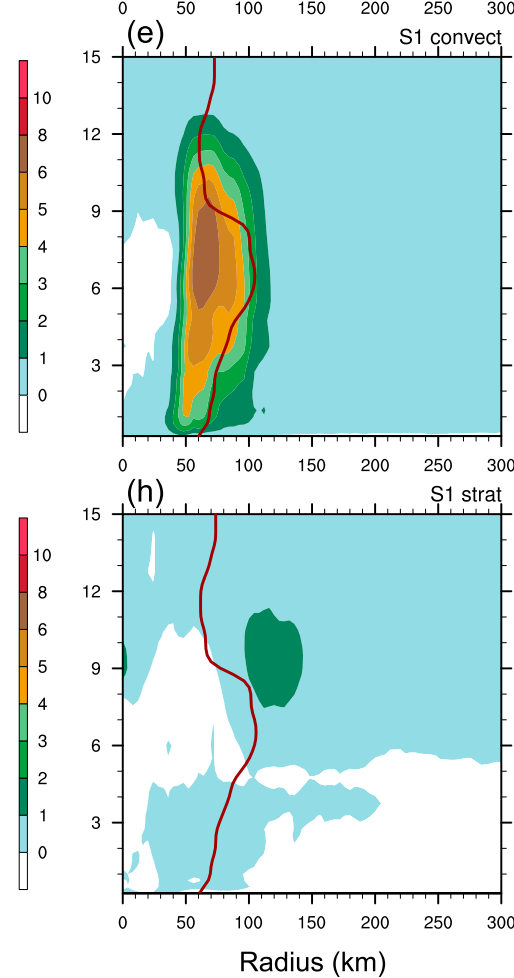
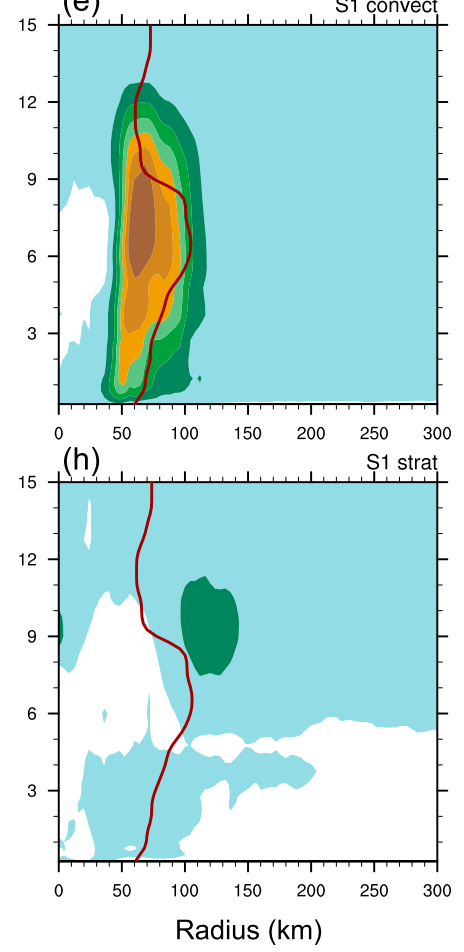

(c)
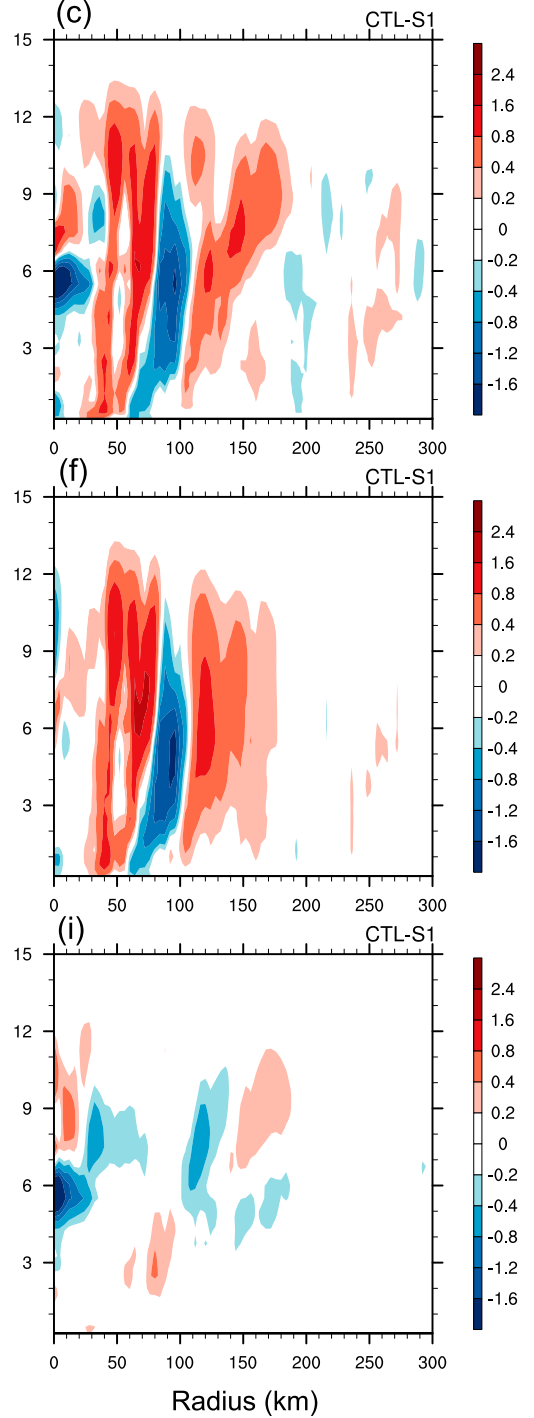

FIG. 12. Composite vertical structure of azimuthal-mean microphysical diabatic heating rate for the (a),(b) total, (d),(e) convective, and (g),(h) stratiform precipitation (shading; $\mathrm{K} \mathrm{h}^{-1}$ ) during the period between 1200 UTC 2 Oct and 0000 UTC 3 Oct for (left) CTL and (center) S1. (c),(f),(i) The differences (i.e., CTL - S1). The thick red lines in the left and center columns denote the mean RMW during the period.

To test this hypothesis, the Sawyer-Eliassen (SE) equation in balanced dynamics (Eliassen 1951) is employed. The $\mathrm{SE}$ equation can diagnose the transverse circulation in response to the axisymmetric diabatic heating and momentum sources. Details of the SE equation are shown in the appendix. To make a direct comparison with Figs. 14a and 14c, Figs. 15a and 15b show the averaged transverse circulations diagnosed by the SE equation with all the momentum and heating forcing terms in the same period. The results indicate that when including all forcing terms, the SE equation generally reproduces the secondary circulation in the WRF simulation well, although the diagnosed upper-level outflow in both experiments shows an overestimation of $\sim 5 \mathrm{~m} \mathrm{~s}^{-1}$ (Figs. 15a and 15b). This is ascribed to the relatively large supergradient winds ${ }^{5}\left(5-6 \mathrm{~m} \mathrm{~s}^{-1}\right.$ outflow; not shown) in the outflow layer, which violates the prerequisite of a gradient wind balance for the SE equation. The SE-derived boundary layer inflow is comparable to the WRF outputs with small errors $\left(\sim 1 \mathrm{~m} \mathrm{~s}^{-1}\right)$, as the simulated TCs prior to RI onset are still of tropical storm intensity and the supergradient winds in the boundary

\footnotetext{
${ }^{5}$ The supergradient winds are calculated by subtracting the radial winds in gradient wind balance from the total radial winds.
} 

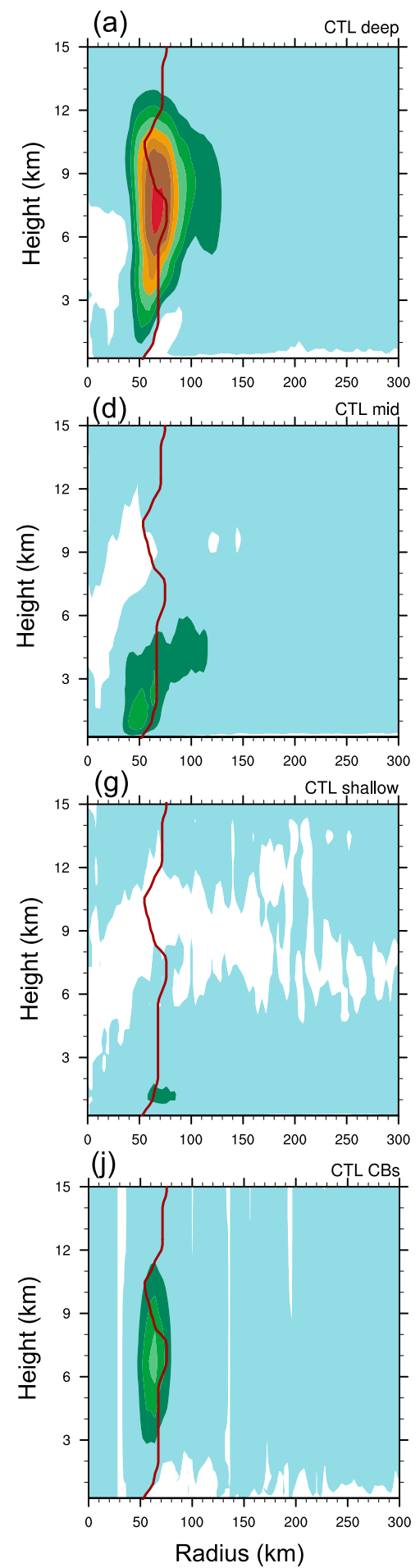

(b)
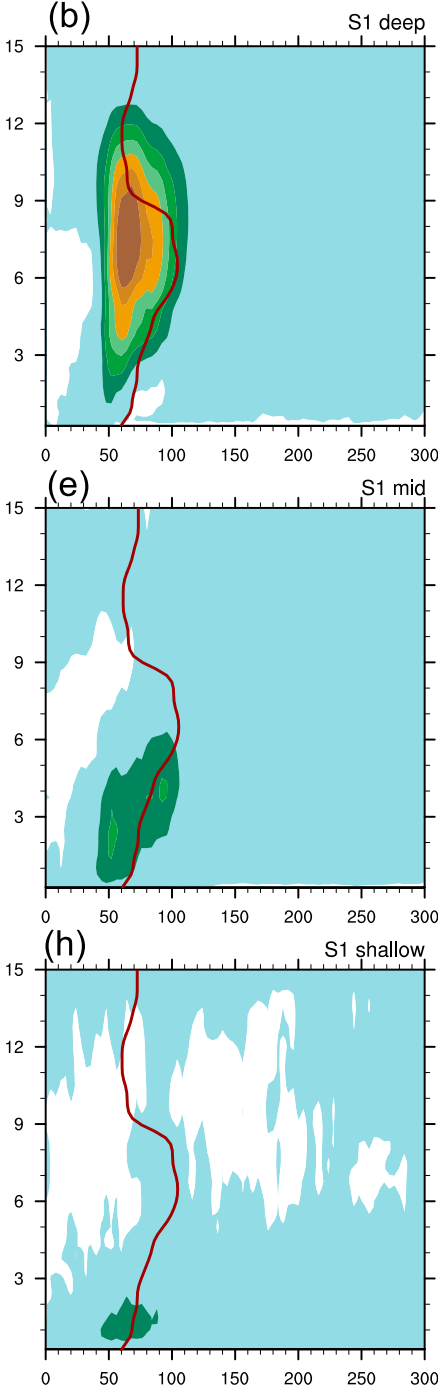

(k)

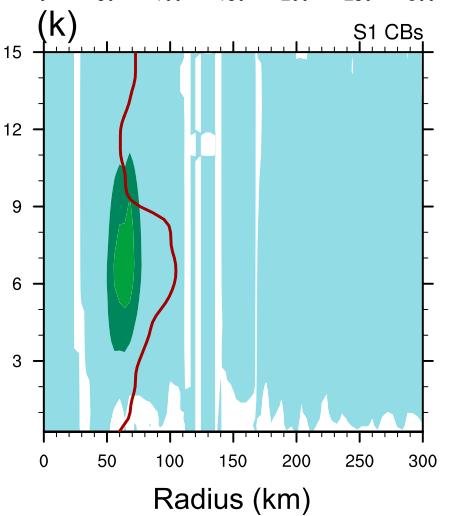

(c)

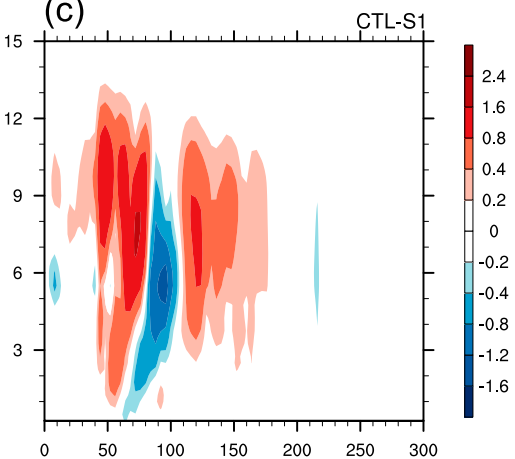

(f)

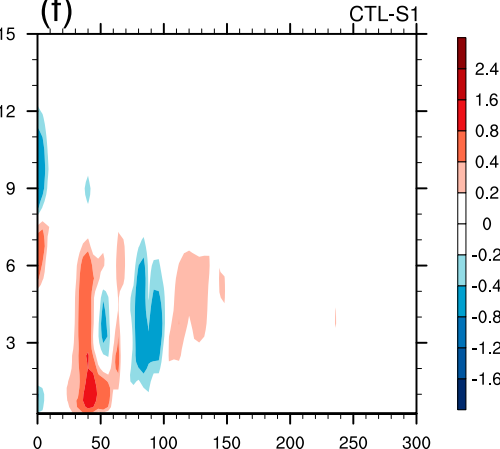

(i)

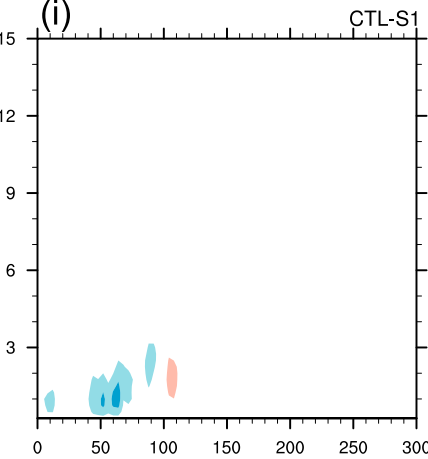

(l)

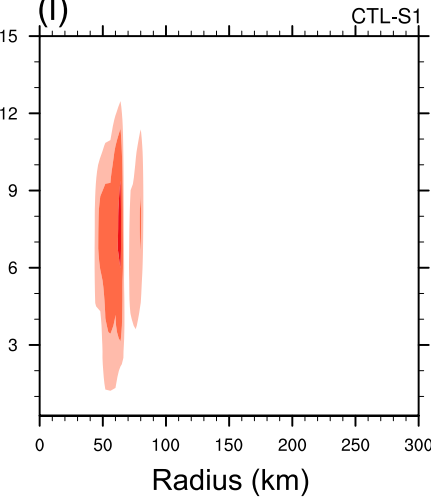

FIG. 13. As in Fig. 12, but for (a)-(c) deep, (d)-(f) midlevel, (g)-(i) shallow convection, and (j)-(l) CBs.

layer are relatively weak $\left(\sim 2 \mathrm{~m} \mathrm{~s}^{-1}\right.$; not shown). The discrepancy in the magnitude of the SE-derived upward branch of the secondary circulation between the CTL and S1 simulations is $\sim 0.05 \mathrm{~m} \mathrm{~s}^{-1}$ (not shown), consistent with the difference in the WRF simulations discussed earlier (Fig. 14e). These results suggest that the SE equation can be employed to further examine the contributions of various forcing terms to the secondary 
(a)
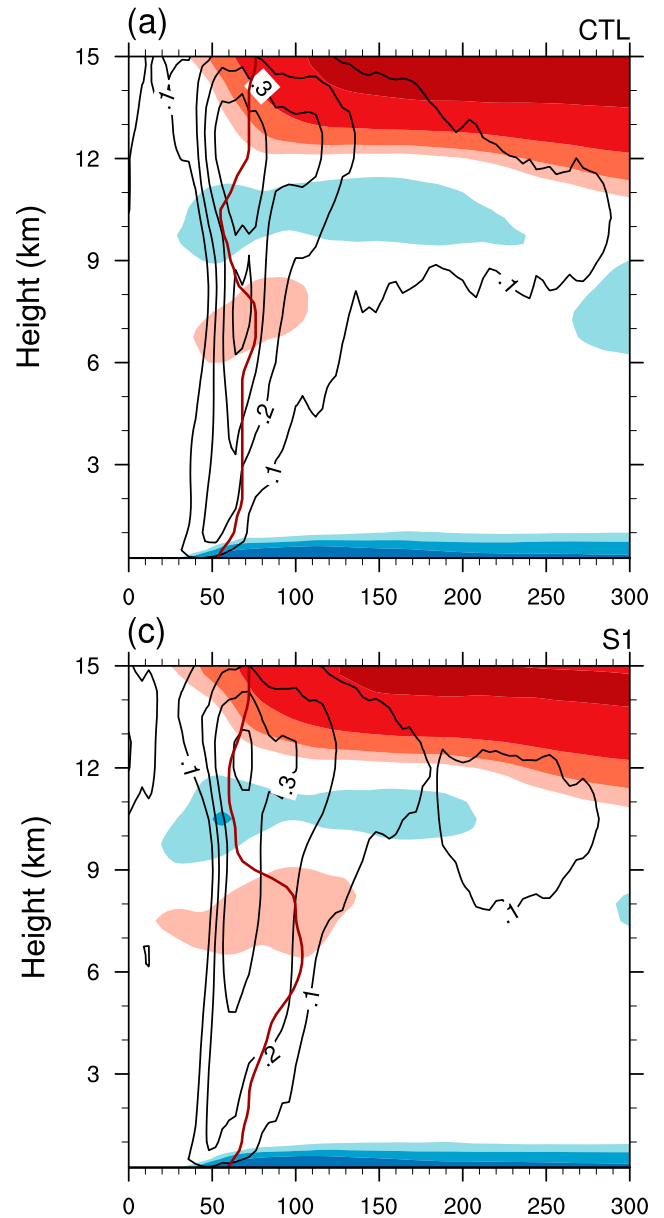

(e)

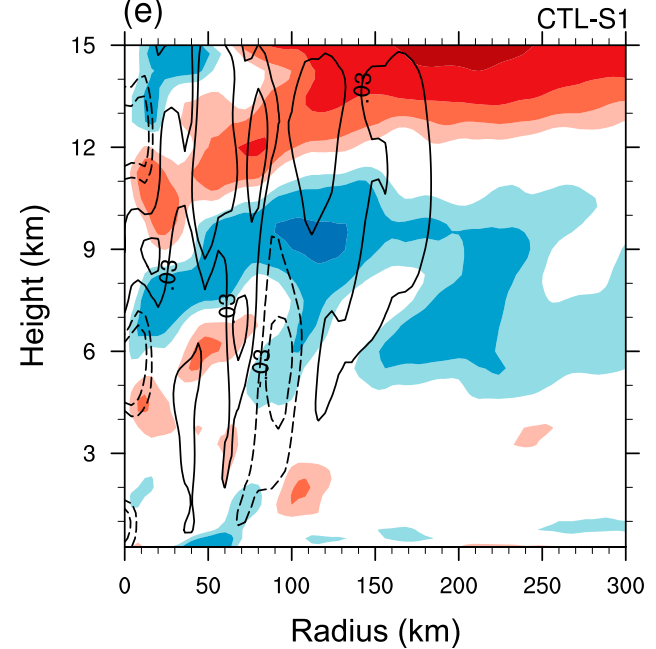

(b)

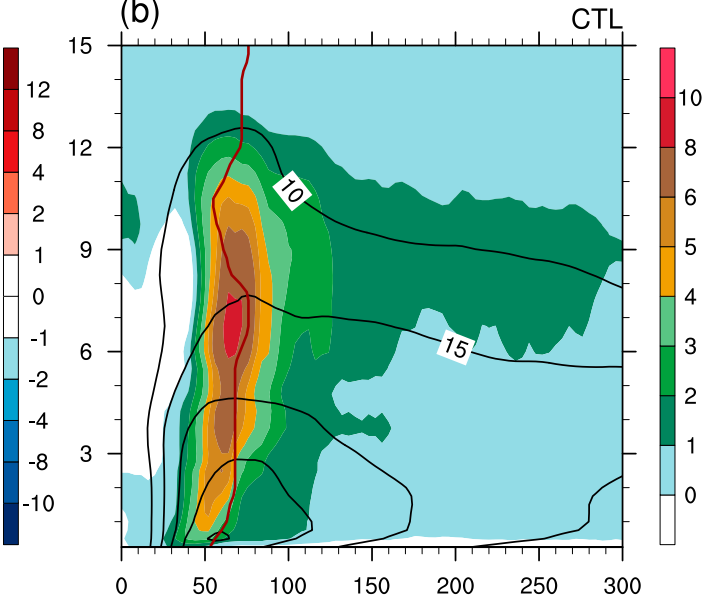

(d)
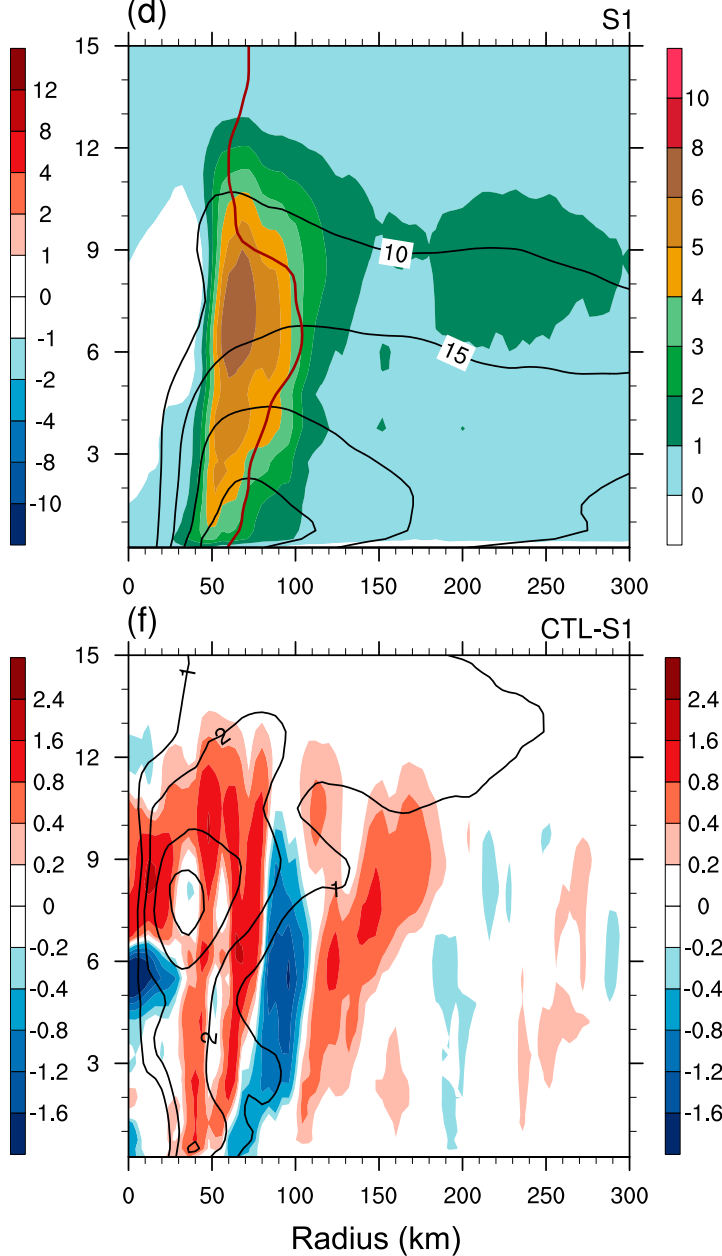

FIG. 14. Composite vertical structure of (a),(c) the azimuthal-mean radial wind (shading; $\mathrm{m} \mathrm{s}^{-1}$ ) and vertical velocity (contours at $0.1,0.2,0.3$, and $0.4 \mathrm{~m} \mathrm{~s}^{-1}$ ), and (b),(d) microphysical diabatic heating (shading; $\mathrm{K} \mathrm{h}^{-1}$ ) and primary circulation (contour interval of $5 \mathrm{~m} \mathrm{~s}^{-1}$ ) during the period between 1200 UTC 2 Oct and 0000 UTC 3 Oct for the (a),(b) CTL and (c),(d) S1 experiments. (e),(f) Their corresponding differences (i.e., CTL - S1). Contours in (e) have the values of $-0.06,-0.03,0.03$, and $0.06 \mathrm{~m} \mathrm{~s}^{-1}$, whereas contours in (f) have values of $1-4 \mathrm{~m} \mathrm{~s}^{-1}$ at a $1 \mathrm{~m} \mathrm{~s}^{-1}$ interval. Thick red lines in (a)-(d) denote the RMW. 

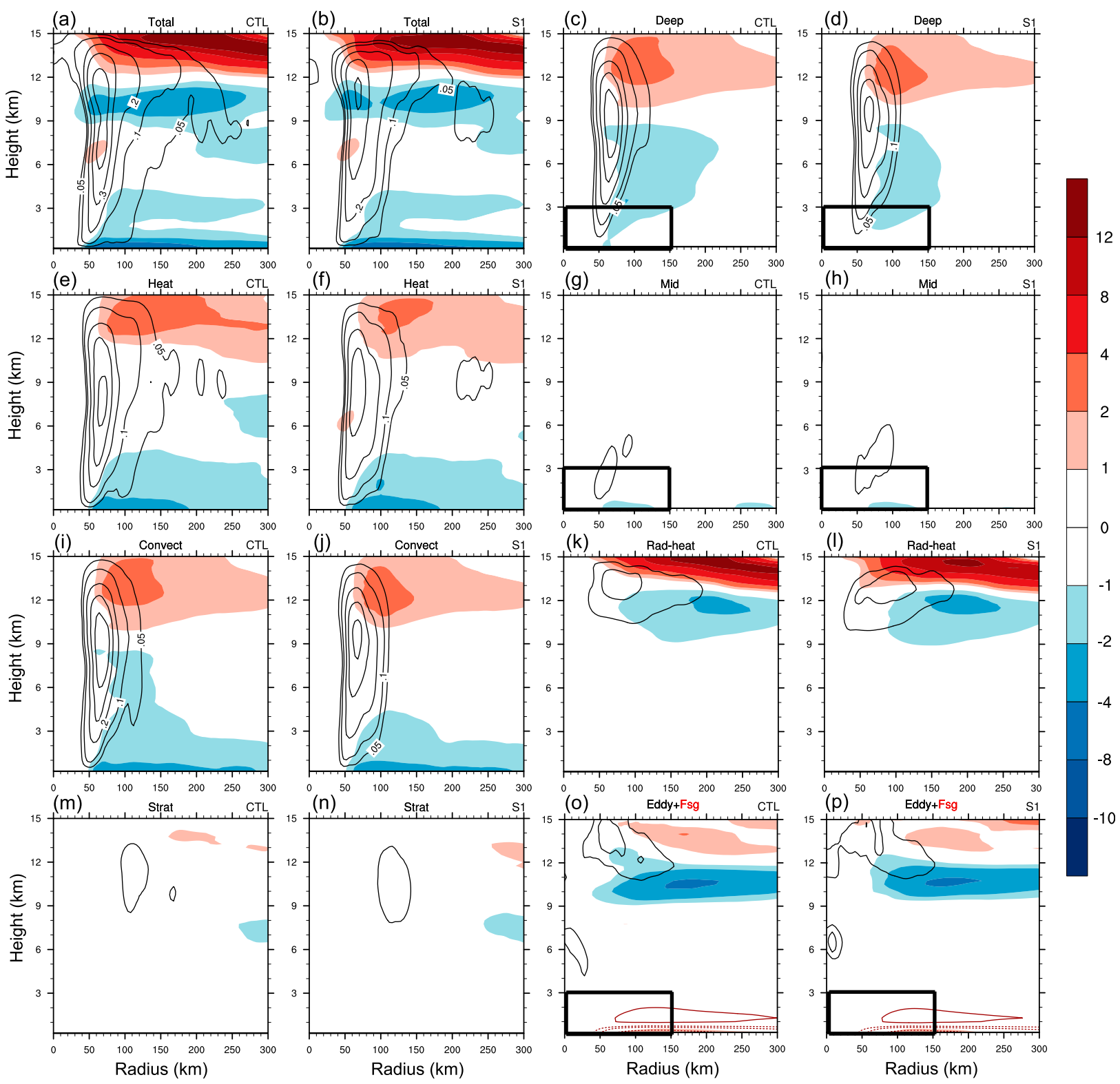

FIG. 15. Vertical structure of azimuthal-mean radial winds (shading; $\mathrm{m} \mathrm{s}^{-1}$ ) and vertical velocity (contours at $0.05,0.1,0.2,0.3$, and $0.4 \mathrm{~m} \mathrm{~s}^{-1}$ ) derived from the SE equation using azimuthal-mean (a),(b) momentum and heating sources; (e),(f) total microphysical diabatic heating; microphysical diabatic heating of (i),(j) convection, (m),(n) stratiform precipitation, (c),(d) deep convection and (g),(h) midlevel convection; and (k),(l) diabatic heating due to radiation; and (o),(p) eddy terms (including heating and momentum) and friction term (radial winds alone; red contours at $-4,-2,-1$ and $0.5 \mathrm{~m} \mathrm{~s}^{-1}$; negative values are dashed). The results are averaged between $1200 \mathrm{UTC}$ 2 Oct and 0000 UTC 3 Oct for (first and third columns) CTL and (second and fourth columns) S1. The black rectangular box denotes the terms that contribute to boundary layer inflow near the RMW.

circulation and to help explain the key factors contributing to the discrepancy in the strength of the secondary circulation between the two experiments.

Figures $15 c-p$ show the SE-derived secondary circulation in response to various forcing terms. The azimuthal-mean radial inflow in the boundary layer is mainly a balanced response to axisymmetric surface friction (Figs. 15o and 15p and Figs. 16e and 16f) as well as the microphysical diabatic heating of the convection (e.g., Figs. 15c-j and 16). The contribution from the diabatic heating resulting from PBL processes is negligible (not shown). The azimuthal-mean diabatic heating of stratiform clouds, primarily outside the RMW $(r=100$ $200 \mathrm{~km}$; Figs. $12 \mathrm{~g}$ and $12 \mathrm{~h}$ ) above the freezing level, 

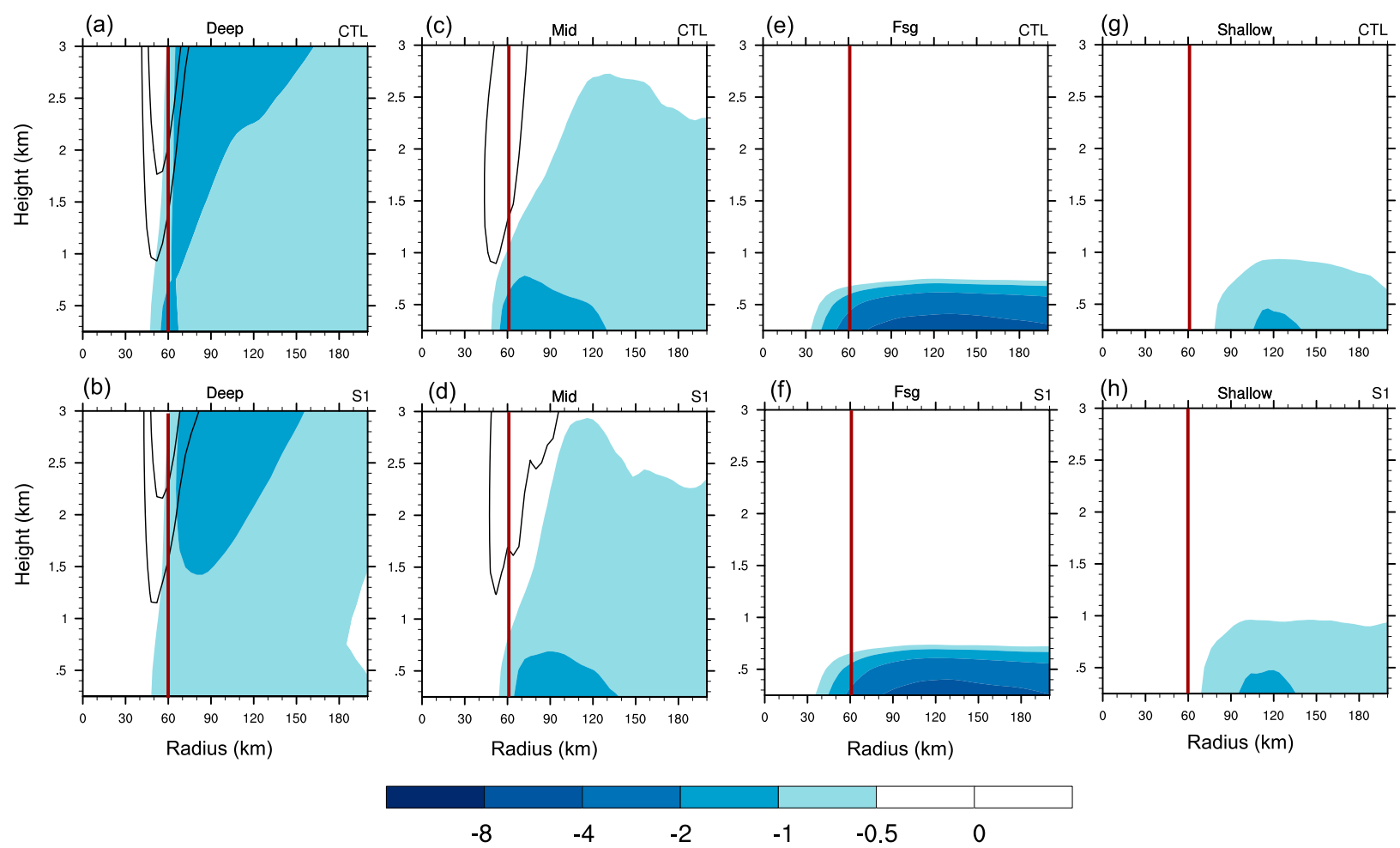

FIG. 16. As in Fig. 15, but zooming in on the lowest $3 \mathrm{~km}$. The SE-derived azimuthal-mean radial inflows using azimuthal-mean diabatic heating of shallow convection are shown in $(\mathrm{g})$ and $(\mathrm{h})$. The red line denotes the reference radius of $60 \mathrm{~km}$.

induces a local secondary circulation in the midtroposphere (Figs. $15 \mathrm{~m}$ and $15 \mathrm{n}$ ). The axisymmetric microphysical diabatic heating of deep and midlevel convection is the major forcing term of the secondary circulation above the boundary layer. In addition, the diabatic heating of radiation and eddy forcing terms (including momentum and heating) enhances the outflow, the inflow beneath, and the upper-tropospheric upward motion (Figs. 15k and $15 \mathrm{l}$ and Figs. 15o and 15p). Their combined effects increase the maximum vertical velocity near the RMW to a height of $12 \mathrm{~km}$ in both experiments.

To explore whether the discrepancies in the boundary layer inflow are responsible for the different timings of RMW contraction, an azimuthal-mean tangential momentum budget equation is employed, as follows:

$$
\frac{\partial \bar{v}}{\partial t}=-\bar{u}(\bar{f}+\bar{\zeta})-\bar{w} \frac{\partial \bar{v}}{\partial z}-\overline{u^{\prime} \zeta^{\prime}}-\overline{w^{\prime} \frac{\partial v^{\prime}}{\partial z}}+\overline{F_{\mathrm{sg}}}
$$

where $u, v$, and $w$ denote storm-relative radial, tangential, and vertical winds, respectively; $f$ and $\zeta$ are the Coriolis parameter and the vertical component of relative vorticity, respectively; and $F_{\mathrm{sg}}$ is the subgridscale tendency of tangential momentum. In Eq. (1), the overbar symbol denotes the azimuthal average and the prime is the deviation from the azimuthal average. The first four terms on the right-hand side are the mean radial flux of absolute mean vertical vorticity (MRAD), the vertical advection of the azimuthal mean tangential wind by the azimuthal-mean vertical motion (MVAD), the radial eddy flux of perturbation vorticity (ERAD), and the vertical advection of asymmetric tangential wind by asymmetric vertical motion (EVAD).

For simplicity, we examine the tangential momentum budget analysis at $250-\mathrm{m}$ height, where the vertical velocity is generally small. Thus, the MVAD is very small compared with the MRAD (not shown). The eddy terms (ERAD and EVAD) are found to be negligible and the friction term reduces the tangential momentum (not shown). The only term that enhances the tangential winds inside the RMW and leads to RMW contraction is the MRAD. Figure $17 \mathrm{~d}$ shows the radial profile of MRAD at $250 \mathrm{~m}$, averaged for the period from 1200 UTC 2 October to 0000 UTC 3 October. The MRAD in CTL is stronger than that in S1, particularly inside $r=50 \mathrm{~km}$. For the CTL TC, the MRAD within $r=40-50 \mathrm{~km}$ is larger than that at $r=60 \mathrm{~km}$ (RMW at 1200 UTC 2 October; Fig. 8b), which is beneficial to RMW contraction. In contrast, the difference in MRAD 
(a)

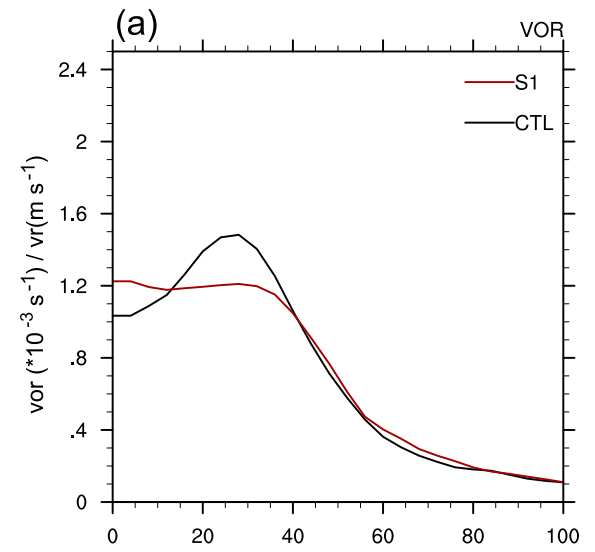

(d)

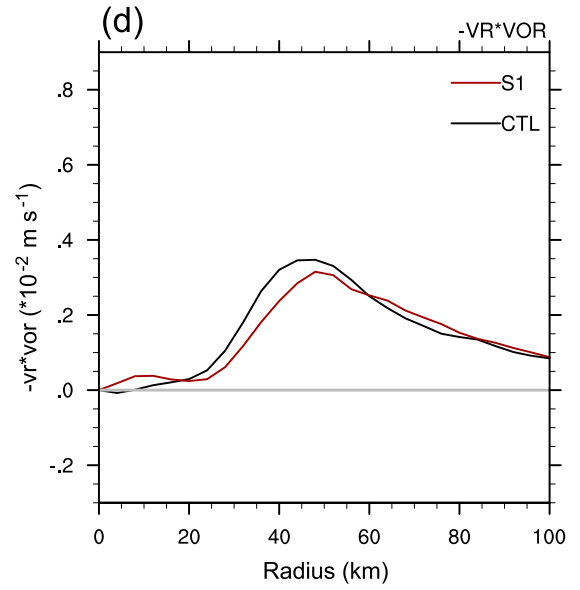

(b)

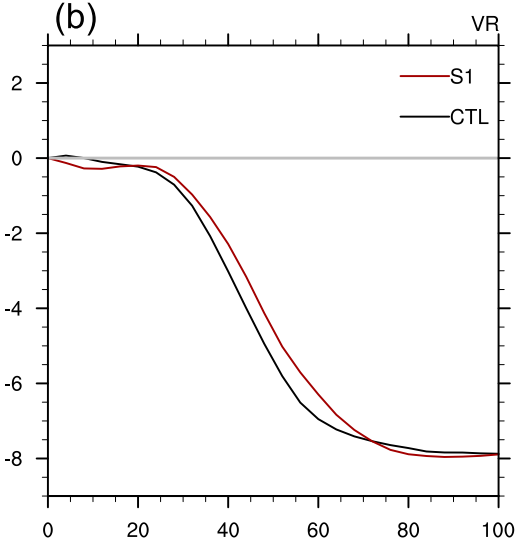

(e)

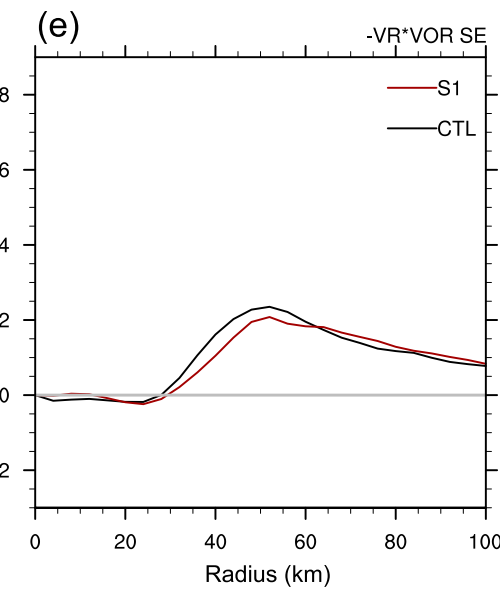

(c)

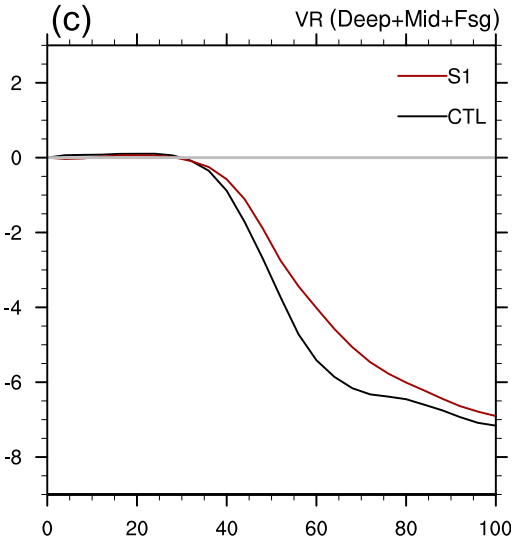

(f)

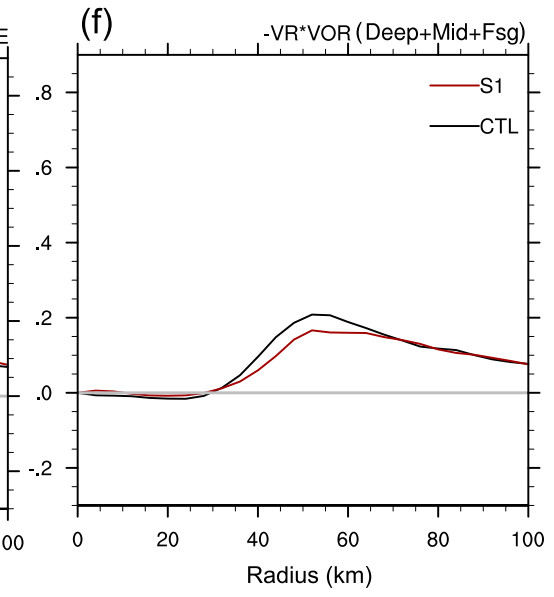

FIG. 17. Mean radial profile of (a) absolute vorticity $\left(10^{-3} \mathrm{~s}^{-1}\right)$, (b) radial winds $\left(\mathrm{m} \mathrm{s}^{-1}\right)$, (c) SE-derived radial winds ( $\left.\mathrm{m} \mathrm{s}^{-1}\right)$ induced by surface friction and the diabatic heating of midlevel and deep convection $\left(F_{\mathrm{sg}}+\right.$ Mid + Deep), and (d) the MRAD (10 $\left.{ }^{-2} \mathrm{~m} \mathrm{~s}^{-1}\right)$ calculated by WRF outputs. (e),(f) As in (d), but using the SE-derived radial inflow with all forcing terms and using only the three forcing terms $\left(F_{\mathrm{sg}}+\mathrm{Mid}+\right.$ Deep $)$, respectively. The averaged period is from 1200 UTC 2 Oct to 0000 UTC 3 Oct.

within $r=40-50$ and $r=60 \mathrm{~km}$ for the $\mathrm{S} 1 \mathrm{TC}$ is much smaller, which may explain the steady RMW of the S1 TC during this period. The stronger MRAD within $r=$ $40-50 \mathrm{~km}$ for the CTL TC is due to the stronger radial inflow ( $\sim 0.8 \mathrm{~m} \mathrm{~s}^{-1}$; Fig. $\left.17 \mathrm{~b}\right)$, as the absolute vorticity in this region is weaker (Fig. 17a).

To understand the extent to which the earlier contraction of lower-level RMW of the CTL TC can be explained by balanced dynamics, we recalculate the MRAD using the SE-derived boundary layer inflow in response to all forcing terms (Fig. 17e). A comparison between Figs. 17d and 17e indicates that the balanced dynamics capture the much faster intensification of tangential momentum within $r=40-50 \mathrm{~km}$ than at $r=$ $60 \mathrm{~km}$ for the CTL TC. However, the peak value of MRAD calculated with SE-derived radial inflows accounts for $\sim 68 \%$ of the true values. In conjunction, the radius of the maximized MRAD in Fig. $17 \mathrm{e}$ is $\sim 6 \mathrm{~km}$ larger than that in Fig. 17d. These results suggest that the unbalanced dynamics in the boundary layer play an additional role in the
RMW contraction of the CTL TC prior to RI onset. The SE-derived boundary layer inflows inside $r=60 \mathrm{~km}$ are induced mainly by diabatic heating of midlevel and deep convection as well as surface friction (the contribution from shallow convection is trivial; see Fig. 16). Using their induced balanced boundary layer inflows (Fig. 17c), the radial profile of MRAD shown in Fig. 17f is comparable to that in Fig. 17e. In short, the balanced dynamics make a significant contribution to the earlier contraction of RMW for the CTL TC, and the most important forcing terms for creating the secondary circulation causing the contraction include the diabatic heating of midlevel and deep convection, and surface friction.

\section{Conclusions and discussion}

\section{a. Summary and conclusions}

Typhoon Mujigae (2015) underwent a notable prelandfall rapid intensification (RI) during its pass over an 
anomalously warm $\left(>29.5^{\circ} \mathrm{C}\right)$ northern South China Sea (SCS) in an environment with moderate VWS. Because of the RI, the maximum sustained $10-\mathrm{m}$ wind increased by $24 \mathrm{~m} \mathrm{~s}^{-1}$ within $30 \mathrm{~h}$, causing an upgrade of Mujigae from a severe tropical storm to a supertyphoon, just prior to landfall. Given this unusual RI event in the northern SCS in October, special attention was paid to the abnormally warm SST relative to the climatological monthly mean.

In this study, triply nested WRF simulations initialized with different SST fields were conducted to investigate the impacts of anomalously warm sea surfaces on the RI of Mujigae. The results indicate that the higher the SST in the northern SCS, the more rapid the intensification and the higher the maximum intensity of the simulated TC. The SST also affects the timing of RI onset, as TCs over cooler sea surfaces begin to RI $13 \mathrm{~h}$ later than those over warmer sea surfaces. By comparing two representative experiments with warmer and cooler SST (i.e., CTL and S1), we examined the key inner-core processes that lead to the differences in RI onset timing.

First, both the CTL and S1 TCs undergo vertical alignment prior to RI onset, and the magnitudes of their vortex tilts are comparable. However, RI onset in CTL occurs $\sim 8 \mathrm{~h}$ before the full vertical alignment (i.e., almost no tilt), while that in S1 TC occurs $\sim 7 \mathrm{~h}$ after. Thus, vertical alignment, while necessary, is insufficient to explain the differences in RI onset timing. Second, as the midlevel TC vortices in both experiments precessed into the upshear flank, the precipitation distribution becomes more azimuthally symmetric. Warmer SST promotes more active convective and stratiform precipitation, leading to higher precipitation symmetricity in the inner-core region preceding the RI onset of the CTL TC. In particular, the stratiform precipitation symmetricity is much higher in CTL than that in S1. Nevertheless, the azimuthal-mean diabatic heating in the inner-core region of both TCs is primarily contributed by midlevel and deep convection. The CTL TC has more midlevel and deep convection near/ inside the RMW and the associated microphysical diabatic heating is much stronger. Our results indicate that it is not simply the presence or even the amount of precipitation but the radial location and the symmetry of precipitation that are more important to RI onset. Third, the earlier RI onset of the CTL TC is closely connected to its earlier near-surface RMW contraction. In fact, the $\mathrm{RI}$ onset of all simulated TCs in the four experiments with different SSTs is well matched to the time when the local Rossby number (Ro) at 10-m height significantly exceeds 12 , which is caused primarily by the contraction of the RMW.

Diagnoses using the Sawyer-Eliassen (SE) equation in balanced dynamics demonstrate that the enhanced microphysical diabatic heating of midlevel and deep convection as well as the strengthened surface friction resulting from more intense TC intensity contribute to stronger boundary layer inflows and larger values of the mean radial flux of absolute mean vertical vorticity (MRAD) inside the RMW, facilitating the earlier RMW contraction and RI onset of the CTL TC.

Finally, we assessed the contribution of unbalanced dynamics in the boundary layer to the earlier RMW contraction of the CTL TC. Our results indicate that the balanced boundary layer inflow induced by the microphysical diabatic heating of midlevel and deep convection as well as the surface friction contribute to $\sim 68 \%$ of the MRAD. The residual (32\%) can be primarily attributed to unbalanced dynamics. Furthermore, the MRAD calculated with the SE-derived inflow peaks at a larger radius $(\sim 6 \mathrm{~km})$ than that calculated with WRF outputs for the CTL TC, suggesting that unbalanced dynamics play an additional role in the earlier RMW contraction of the CTL TC. These findings support the statement in Smith and Montgomery (2015) that frictional driven inflow or unbalanced dynamics in the boundary layer is an indispensable component to spin up the TC circulation.

\section{b. Additional discussion}

Precursors or indicators of RI in terms of inner-core processes in the environments with moderate VWS have been a topic of debate in the TC community for years. Many of the precursors occur simultaneously near RI onset-for example, the increased precipitation symmetry and vertical alignment (e.g., Tao and Zhang 2014; Rogers et al. 2015; Munsell et al. 2017; Chen et al. 2018)—which imped our understanding as to which of these precursors are more predominant. In a set of numerical simulations of Typhoon Mujigae using different SSTs, some of these precursors were decoupled, allowing us to identify the more immediate cause of RI. We found that besides vertical alignment, a more effective RI indicator is the formation of a compact/strong TC inner core (i.e., large Ro) with high precipitation symmetry.

One caution of this study is that the identified RI onset indicator applicable to different SST conditions, namely, Ro $>12$ at 10-m height, cannot be overgeneralized beyond this case. We should be aware that the value of Ro is latitude dependent as a result of the inclusion of the Coriolis parameter. Miyamoto and Nolan (2018) noted that, through a large ensemble of idealized simulations, the value of Ro at the RI onset can spread over a wide range by changing the size and intensity of the initial vortex, translational speed, and VWS magnitude, although they calculated the Ro at 2-km height and adopted a different criteria of RI onset. 
Nevertheless, this study highlights the importance of inner-core structure and intensity to RI onset timing, in agreement with the results of idealized (Miyamoto and Takemi 2015; Miyamoto and Nolan 2018) and real-case simulation studies (Chang and Wu 2017), and statistical studies (e.g., Carrasco et al. 2014; Xu and Wang 2015). Meanwhile, our results also help reconcile some of the discrepancies in interpreting the importance of different modes of precipitation by pointing out a synergistic relationship between different types of precipitation prior to RI onset. Although the TC going to RI (i.e., the CTL TC) has a larger area coverage of precipitation primarily because of more stratiform precipitation, it is the midlevel and deep convection (including CBs) that dominate the azimuthal-mean diabatic heating. This fact does not devalue the role of stratiform precipitation preceding RI onset. Rather, we recognize that the CTL TC has more stratiform downwind of the convective precipitation in the upshear flank (e.g., Figs. 7 and 9). It is hypothesized that the stratiform precipitation may play an important role in moistening the upshear flank and thus help the sustainment of more active midlevel and deep convection in the upshear flank during the precession of the midlevel TC vortex. In the future detailed analyses of the various types of precipitation in the shear-relative quadrants will be conducted.

As a closing note, it is worth mentioning how the TCs evolve from an asymmetric to a symmetric structure prior to RI onset in an essentially three-dimensional rather than axisymmetric formulation (e.g., Rogers et al. 2015; Chen et al. 2017; Leighton et al. 2018). What factors promote or inhibit the precipitation symmetrization? Is the earlier RMW contraction facilitated by a more symmetric TC vortex? These open questions are beyond the scope of this study, while addressing them can further enhance our understanding in the dynamics leading to RI onset in the environments with moderate VWS. More observational and numerical simulation studies are required to answer these questions.

Acknowledgments. The authors thank three anonymous reviewers for their perceptive comments on the original manuscript. This study had been supported by the National Key R\&D Program of China under Grant 2017YFC1501601; the Natural Science Foundation of China Grants 41730965, 41605033, 41475046, and 41730960; the National Foundational Research "973" Program of China (2013CB430103); and the Fundamental Research Funds for the Central Universities. The authors are grateful for the helpful discussions with Drs. Robert Rogers, Xiaodong Tang, Yuqing Wang, and Jian-Feng Gu.

\section{APPENDIX}

\section{Sawyer-Eliassen Equation}

With the Boussinesq approximation and the constraint of thermal wind balance, the Sawyer-Eliassen (SE) equation for the transverse streamfunction is shown as follows (Montgomery et al. 2006):

$\frac{\partial}{\partial r}\left(\frac{A}{r} \frac{\partial \bar{\psi}}{\partial r}+\frac{B}{r} \frac{\partial \bar{\psi}}{\partial z}\right)+\frac{\partial}{\partial z}\left(\frac{C}{r} \frac{\partial \bar{\psi}}{\partial z}+\frac{B}{r} \frac{\partial \bar{\psi}}{\partial r}\right)=\frac{\partial \bar{Q}}{\partial r}-\frac{\partial(\bar{\xi} \bar{F})}{\partial z}$

where $r$ and $z$ represent the radius and pseudoheight, respectively. The overbar symbol denotes the azimuthal average. The transverse streamfunction $\bar{\psi}$ is related to the azimuthal-mean radial and vertical velocity by $\bar{u}=-(1 / r)(\partial \bar{\psi} / \partial z)$ and $\bar{w}=(1 / r)(\partial \bar{\psi} / \partial r)$, respectively. The $A, B$, and $C$ are static stability, baroclinity, and inertial stability, respectively. They are given as

$$
\begin{aligned}
& A=\overline{N^{2}}=\frac{g}{\theta_{0}} \frac{\partial \bar{\theta}}{\partial z}, \\
& B=-\bar{\xi} \frac{\partial \bar{v}}{\partial z}, \\
& C=\bar{\xi} \bar{\eta},
\end{aligned}
$$

where $\bar{\theta}, \bar{v}$, and $\bar{\eta}$ are mean potential temperature, tangential wind, and absolute vertical vorticity, respectively; and $\bar{\xi}=f_{0}+2 \bar{v} / r$ is the local Coriolis parameter. The heating and momentum sources on the rhs of Eq. (A1) are defined as

$$
\begin{aligned}
& \bar{Q}=\frac{g}{\theta_{0}}\left(-\overline{V_{r}^{\prime} \frac{\partial \theta^{\prime}}{\partial r}}-\overline{w^{\prime} \frac{\partial \theta^{\prime}}{\partial z}}+\overline{\dot{\theta}}\right), \\
& \bar{F}=-\overline{V_{r}^{\prime} \zeta^{\prime}}-\overline{w^{\prime} \frac{\partial v^{\prime}}{\partial z}}+\overline{F_{\mathrm{sg}}},
\end{aligned}
$$

where the prime is the deviation from the azimuthal average. The first two terms in both sources represent the mean eddy terms, $\bar{\theta}$ is the mean diabatic heating rate (resulting from PBL, radiation, and microphysics processes) in the inner model domains; and $\bar{F}_{\mathrm{sg}}$ is the mean subgrid-scale tendency of tangential momentum. Given the structure of vortex (i.e., $A, B$, and $C$ ) and the heating and momentum sources, we can solve this equation and get the transverse circulation, if the elliptic condition $A C-B^{2}>0$ is satisfied and proper boundary conditions are specified. For more details of the SE equation and the specific calculation procedures, we will refer interested readers to Montgomery et al. (2006). 


\section{REFERENCES}

Alvey, G. R., III, J. Zawislak, and E. Zipser, 2015: Precipitation properties observed during tropical cyclone intensity change. Mon. Wea. Rev., 143, 4476-4492, https://doi.org/10.1175/ MWR-D-15-0065.1.

Carrasco, C. A., C. W. Landsea, and Y.-L. Lin, 2014: The influence of tropical cyclone size on its intensification. Wea. Forecasting, 29, 582-590, https://doi.org/10.1175/WAF-D-13-00092.1.

Chang, C.-C., and C.-C. Wu, 2017: On the processes leading to the rapid intensification of Typhoon Megi (2010). J. Atmos. Sci., 74, 1169-1200, https://doi.org/10.1175/JAS-D-16-0075.1.

Chen, H., and D.-L. Zhang, 2013: On the rapid intensification of Hurricane Wilma (2005). Part II: Convective bursts and the upper-level warm core. J. Atmos. Sci., 70, 146-162, https:// doi.org/10.1175/JAS-D-12-062.1.

__ and S. G. Gopalakrishnan, 2015: A study on the asymmetric rapid intensification of Hurricane Earl (2010) using the HWRF system. J. Atmos. Sci., 72, 531-550, https://doi.org/ 10.1175/JAS-D-14-0097.1.

Chen, X., Y. Wang, and K. Zhao, 2015: Synoptic flow patterns and large-scale characteristics associated with rapidly intensifying tropical cyclones in the South China Sea. Mon. Wea. Rev., 143, 64-87, https://doi.org/10.1175/MWR-D-13-00338.1.

,,-- , and D. Wu, 2017: A numerical study on rapid intensification of Typhoon Vicente (2012) in the South China Sea. Part I: Verification of simulation, storm-scale evolution, and environmental contribution. Mon. Wea. Rev., 145, 877898, https://doi.org/10.1175/MWR-D-16-0147.1.

,-- J. Fang, and M. Xue, 2018: A numerical study on rapid intensification of Typhoon Vicente (2012) in the South China Sea. Part II: Roles of inner-core processes. J. Atmos. Sci., 75, 235-255, https://doi.org/10.1175/JAS-D-17-0129.1.

CMA, 2015: Yearbook of Tropical Cyclone (in Chinese). China Meteorological Press, $156 \mathrm{pp}$.

Corbosiero, K. L., and J. Molinari, 2002: The effects of vertical wind shear on the distribution of convection in tropical cyclones. Mon. Wea. Rev., 130, 2110-2123, https://doi.org/ 10.1175/1520-0493(2002)130<2110:TEOVWS>2.0.CO;2.

Črnivec, N., R. K. Smith, and G. Kilroy, 2016: Dependence of tropical cyclone intensification rate on sea-surface temperature. Quart. J. Roy. Meteor. Soc., 142, 1618-1627, https:// doi.org/10.1002/qj.2752.

DeMaria, M., and J. Kaplan, 1994: A Statistical Hurricane Intensity Prediction Scheme (SHIPS) for the Atlantic basin. Wea. Forecasting, 9, 209-220, https://doi.org/10.1175/1520-0434 (1994)009<0209:ASHIPS>2.0.CO;2.

— C. R. Sampson, J. A. Knaff, and K. D. Musgrave, 2014: Is tropical cyclone intensity guidance improving? Bull. Amer. Meteor. Soc., 95, 387-398, https://doi.org/10.1175/BAMS-D12-00240.1.

Dudhia, J., 1989: Numerical study of convection observed during the Winter Monsoon Experiment using a mesoscale twodimensional model. J. Atmos. Sci., 46, 3077-3107, https:// doi.org/10.1175/1520-0469(1989)046<3077:NSOCOD > 2.0.CO;2.

Eliassen, A., 1951: Slow thermally or frictionally controlled meridional circulation in a circular vortex. Astrophys. Norv., 5, 19.

Emanuel, K., 2017: Will global warming make hurricane forecasting more difficult? Bull. Amer. Meteor. Soc., 98, 495-501, https://doi.org/10.1175/BAMS-D-16-0134.1.

Fritz, C., Z. Wang, S. W. Nesbitt, and T. J. Dunkerton, 2016: Vertical structure and contribution of different types of precipitation during Atlantic tropical cyclone formation as revealed by
TRMM PR. Geophys. Res. Lett., 43, 894-901, https://doi.org/ 10.1002/2015GL067122.

Harnos, D. S., and S. W. Nesbitt, 2011: Convective structure in rapidly intensifying tropical cyclones as depicted by passive microwave measurements. Geophys. Res. Lett., 38, L07805, https://doi.org/10.1029/2011GL047010.

— clone rapid intensification. Part II: Vertical motion and cloud populations. Quart. J. Roy. Meteor. Soc., 142, 1832-1846, https://doi.org/10.1002/qj.2778.

Hazelton, A. T., R. E. Hart, and R. F. Rogers, 2017: Analyzing simulated convective bursts in two Atlantic hurricanes. Part II: Intensity change due to bursts. Mon. Wea. Rev., 145, 30953117, https://doi.org/10.1175/MWR-D-16-0268.1.

Hendricks, E. A., M. S. Peng, B. Fu, and T. Li, 2010: Quantifying environmental control on tropical cyclone intensity change. Mon. Wea. Rev., 138, 3243-3271, https://doi.org/10.1175/ 2010MWR3185.1.

Houze, R. A., W.-C. Lee, and M. M. Bell, 2009: Convective contribution to the genesis of Hurricane Ophelia (2005). Mon. Wea. Rev., 137, 2778-2800, https://doi.org/10.1175/ 2009MWR2727.1.

Janjić, Z. I., 1990: The step-mountain coordinate: Physical package. Mon. Wea. Rev., 118, 1429-1443, https://doi.org/10.1175/15200493(1990)118<1429:TSMCPP > 2.0.CO;2.

Jones, S. C., 1995: The evolution of vortices in vertical shear. I: Initially barotropic vortices. Quart. J. Roy. Meteor. Soc., 121, 821-851, https://doi.org/10.1002/qj.49712152406.

Kain, J. S., and J. M. Fritsch, 1993: Convective parameterization for mesoscale models: The Kain-Fritsch scheme. The Representation of Cumulus Convection in Numerical Models, K. A. Emanuel and D. J. Raymond, Eds., Springer, 165-170, https:// doi.org/10.1007/978-1-935704-13-3_16.

Kaplan, J., and M. DeMaria, 2003: Large-scale characteristics of rapidly intensifying tropical cyclones in the North Atlantic basin. Wea. Forecasting, 18, 1093-1108, https://doi.org/ 10.1175/1520-0434(2003)018<1093:LCORIT > 2.0.CO;2.

,$- \ldots$, and J. A. Knaff, 2010: A revised tropical cyclone rapid intensification index for the Atlantic and eastern North Pacific basins. Wea. Forecasting, 25, 220-241, https://doi.org/10.1175/ 2009WAF2222280.1.

Kieper, M. E., and H. Jiang, 2012: Predicting tropical cyclone rapid intensification using the $37 \mathrm{GHz}$ ring pattern identified from passive microwave measurements. Geophys. Res. Lett., 39, L13804, https://doi.org/10.1029/2012GL052115.

LeComte, D., 2016: International weather highlights 2015: Historic El Niño, record warmth, record hurricanes, lower damages. Weatherwise, 69, 20-27, https://doi.org/10.1080/ 00431672.2016 .1159487$.

Leighton, H., S. Gopalakrishnan, J. A. Zhang, R. F. Rogers, Z. Zhang, and V. Tallapragada, 2018: Azimuthal distribution of deep convection, environmental factors, and tropical cyclone rapid intensification: A perspective from HWRF ensemble forecasts of Hurricane Edouard (2014). J. Atmos. Sci., 75, 275-295, https://doi.org/10.1175/JAS-D-17-0171.1.

McFarquhar, G. M., B. F. Jewett, M. S. Gilmore, S. W. Nesbitt, and T.-L. Hsieh, 2012: Vertical velocity and microphysical distributions related to rapid intensification in a simulation of Hurricane Dennis (2005). J. Atmos. Sci., 69, 3515-3534, https:// doi.org/10.1175/JAS-D-12-016.1.

Mellor, G. L., and T. Yamada, 1982: Development of a turbulence closure model for geophysical fluid problems. Rev. Geophys., 20, 851-875, https://doi.org/10.1029/RG020i004p00851. 
Miyamoto, Y., and T. Takemi, 2015: A triggering mechanism for rapid intensification of tropical cyclones. J. Atmos. Sci., 72, 2666-2681, https://doi.org/10.1175/JAS-D-14-0193.1.

— intensification in tropical cyclones as shown in a large ensemble of idealized simulations. J. Atmos. Sci., 75, 555-569, https://doi.org/10.1175/JAS-D-17-0177.1.

Mlawer, E. J., S. J. Taubman, P. D. Brown, M. J. Iacono, and S. A. Clough, 1997: Radiative transfer for inhomogeneous atmospheres: RRTM, a validated correlated-k model for the longwave. J. Geophys. Res., 102,16663-16682, https://doi.org/ 10.1029/97JD00237.

Molinari, J., and D. Vollaro, 2010: Rapid intensification of a sheared tropical storm. Mon. Wea. Rev., 138, 3869-3885, https://doi.org/10.1175/2010MWR3378.1.

Montgomery, M. T., M. E. Nicholls, T. A. Cram, and A. B. Saunders, 2006: A vortical hot tower route to tropical cyclogenesis. J. Atmos. Sci., 63, 355-386, https://doi.org/10.1175/JAS3604.1.

Munsell, E. B., F. Zhang, J. A. Sippel, S. A. Braun, and Y. Weng, 2017: Dynamics and predictability of the intensification of Hurricane Edouard (2014). J. Atmos. Sci., 74, 573-595, https:// doi.org/10.1175/JAS-D-16-0018.1.

Nguyen, L. T., J. Molinari, and D. Thomas, 2014: Evaluation of tropical cyclone center identification methods in numerical models. Mon. Wea. Rev., 142, 4326-4339, https://doi.org/ 10.1175/MWR-D-14-00044.1.

Pendergrass, A. G., and H. E. Willoughby, 2009: Diabatically induced secondary flows in tropical cyclones. Part I: Quasisteady forcing. Mon. Wea. Rev., 137, 805-821, https://doi.org/ 10.1175/2008MWR2657.1.

Rappin, E. D., and D. S. Nolan, 2012: The effect of vertical shear orientation on tropical cyclogenesis. Quart. J. Roy. Meteor. Soc., 138, 1035-1054, https://doi.org/10.1002/qj.977.

Reasor, P. D., and M. T. Montgomery, 2015: Evaluation of a heuristic model for tropical cyclone resilience. J. Atmos. Sci., 72, 1765-1782, https://doi.org/10.1175/JAS-D-14-0318.1.

_ - M. D. Eastin, and J. F. Gamache, 2009: Rapidly intensifying Hurricane Guillermo (1997). Part I: Low-wavenumber structure and evolution. Mon. Wea. Rev., 137, 603-631, https://doi.org/ 10.1175/2008MWR2487.1.

Rios-Berrios, R., and R. D. Torn, 2017: Climatological analysis of tropical cyclone intensity changes under moderate vertical wind shear. Mon. Wea. Rev., 145, 1717-1738, https://doi.org/ 10.1175/MWR-D-16-0350.1.

Rogers, R., 2010: Convective-scale structure and evolution during a high-resolution simulation of tropical cyclone rapid intensification. J. Atmos. Sci., 67, 44-70, https://doi.org/10.1175/ 2009JAS3122.1.

_- P. Reasor, and S. Lorsolo, 2013: Airborne Doppler observations of the inner-core structural differences between intensifying and steady-state tropical cyclones. Mon. Wea. Rev., 141, 2970-2991, https://doi.org/10.1175/MWR-D-12-00357.1.

- _ - and J. A. Zhang, 2015: Multiscale structure and evolution of Hurricane Earl (2010) during rapid intensification. Mon. Wea. Rev., 143, 536-562, https://doi.org/10.1175/ MWR-D-14-00175.1.

_ J. A. Zhang, J. Zawislak, H. Jiang, G. R. Alvey, E. J. Zipser, and S. N. Stevenson, 2016: Observations of the structure and evolution of Hurricane Edouard (2014) during intensity change. Part II: Kinematic structure and the distribution of deep convection. Mon. Wea. Rev., 144, 3355-3376, https://doi.org/ 10.1175/MWR-D-16-0017.1.

Schubert, W. H., and J. J. Hack, 1982: Inertial stability and tropical cyclone development. J. Atmos. Sci., 39, 1687-1697, https://doi.org/ 10.1175/1520-0469(1982)039<1687:ISATCD > 2.0.CO;2.

Skamarock, W. C., J. B. Klemp, J. Dudhia, D. O. Gill, D. M. Barker, W. Wang, and J. G. Powers, 2008: A description of the Advanced Research WRF version 3. NCAR Tech Note NCAR/ TN-475+STR, 113 pp., https://doi.org/10.5065/D68S4MVH.

Smith, R. K., and M. T. Montgomery, 2015: Toward clarity on understanding tropical cyclone intensification. J. Atmos. Sci., 72, 3020-3031, https://doi.org/10.1175/JAS-D-15-0017.1.

Stern, D. P., J. L. Vigh, D. S. Nolan, and F. Zhang, 2015: Revisiting the relationship between eyewall contraction and intensification. J. Atmos. Sci., 72, 1283-1306, https://doi.org/10.1175/JAS-D14-0261.1.

Stevenson, S. N., K. L. Corbosiero, and J. Molinari, 2014: The convective evolution and rapid intensification of Hurricane Earl (2010). Mon. Wea. Rev., 142, 4364-4380, https://doi.org/ 10.1175/MWR-D-14-00078.1.

Susca-Lopata, G., J. Zawislak, E. J. Zipser, and R. F. Rogers, 2015: The role of observed environmental conditions and precipitation evolution in the rapid intensification of Hurricane Earl (2010). Mon. Wea. Rev., 143, 2207-2223, https://doi.org/ 10.1175/MWR-D-14-00283.1.

Tao, C., and H. Jiang, 2015: Distributions of shallow to very deep precipitation-convection in rapidly intensifying tropical cyclones. J. Climate, 28, 8791-8824, https://doi.org/10.1175/JCLID-14-00448.1.

and J. Zawislak, 2017: The relative importance of stratiform and convective rainfall in rapidly intensifying tropical cyclones. Mon. Wea. Rev., 145, 795-809, https://doi.org/10.1175/ MWR-D-16-0316.1.

Tao, D., and F. Zhang, 2014: Effect of environmental shear, seasurface temperature, and ambient moisture on the formation and predictability of tropical cyclones: An ensemble-mean perspective. J. Adv. Model. Earth Syst., 6, 384-404, https:// doi.org/10.1002/2014MS000314.

Thompson, G., P. R. Field, R. M. Rasmussen, and W. D. Hall, 2008: Explicit forecasts of winter precipitation using an improved bulk microphysics scheme. Part II: Implementation of a new snow parameterization. Mon. Wea. Rev., 136, 5095-5115, https://doi.org/10.1175/2008MWR2387.1.

Wang, H., and Y. Wang, 2014: A numerical study of Typhoon Megi (2010). Part I: Rapid intensification. Mon. Wea. Rev., 142, 2948, https://doi.org/10.1175/MWR-D-13-00070.1.

Wang, Y., and G. J. Holland, 1996: Tropical cyclone motion and evolution in vertical shear. J. Atmos. Sci., 53, 3313-3332, https://doi.org/10.1175/1520-0469(1996)053<3313:TCMAEI > 2.0.CO;2

$\mathrm{Xu}$, J., and Y. Wang, 2015: A statistical analysis on the dependence of tropical cyclone intensification rate on the storm intensity and size in the North Atlantic. Wea. Forecasting, 30, 692-701, https://doi.org/10.1175/WAF-D-14-00141.1.

Zagrodnik, J. P., and H. Jiang, 2014: Rainfall, convection, and latent heating distributions in rapidly intensifying tropical cyclones. J. Atmos. Sci., 71, 2789-2809, https://doi.org/10.1175/ JAS-D-13-0314.1.

Zhang, F., and D. Tao, 2013: Effects of vertical wind shear on the predictability of tropical cyclones. J. Atmos. Sci., 70, 975-983, https://doi.org/10.1175/JAS-D-12-0133.1. 